\title{
A Momentum-Conserving Implicit Material Point Method for Surface Tension with Contact Angles and Spatial Gradients
}

\author{
JINGYU CHEN, UCLA, USA \\ VICTORIA KALA, UCLA, USA \\ ALAN MARQUEZ-RAZON, UCLA, USA \\ ELIAS GUEIDON, UCLA, USA \\ DAVID A. B. HYDE, UCLA, USA \\ JOSEPH TERAN, UC Davis, USA
}

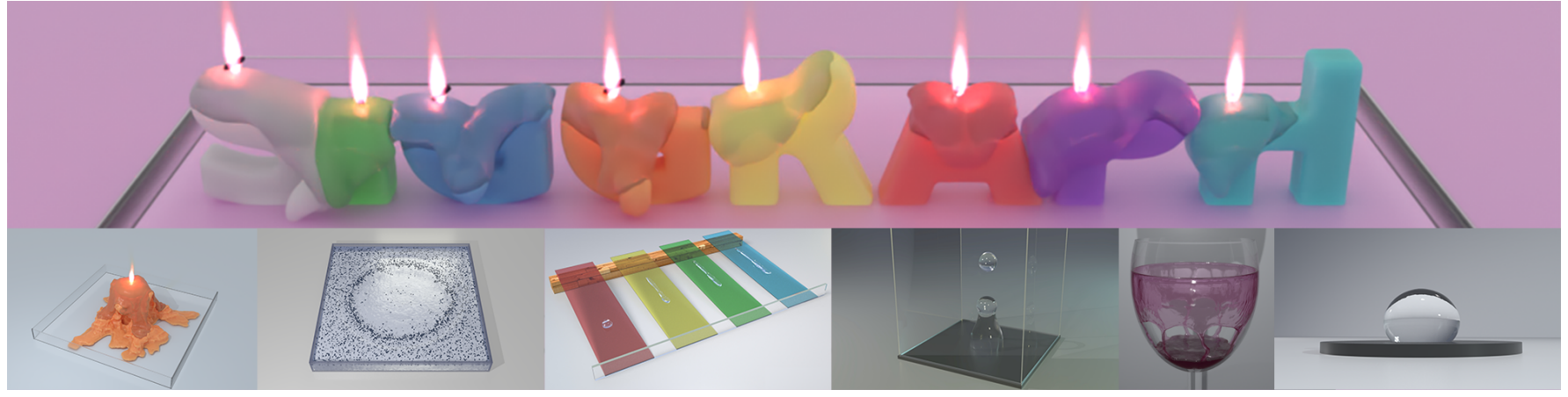

Fig. 1. Our method enables the simulation of a wide variety of thermomechanical and surface-tension-driven effects. (Top) Letter-shaped candles melt and interact. (Bottom) A large melting candle; soap spreading on a water surface; water droplets falling and streaking on ramps; partial rebound of a water droplet impact; wine settling in a glass; a water droplet settling on a hydrophobic surface.

We present a novel Material Point Method (MPM) discretization of surface tension forces that arise from spatially varying surface energies. These variations typically arise from surface energy dependence on temperature and/or concentration. Furthermore, since the surface energy is an interfacial property depending on the types of materials on either side of an interface, spatial variation is required for modeling the contact angle at the triple junction between a liquid, solid and surrounding air. Our discretization is based on the surface energy itself, rather than on the associated traction condition most commonly used for discretization with particle methods. Our energy based approach automatically captures surface gradients without the explicit need to resolve them as in traction condition based approaches. We include an implicit discretization of thermomechanical material coupling with a novel particle-based enforcement of Robin boundary conditions associated with convective heating. Lastly, we design a particle resampling approach needed to achieve perfect conservation of linear and angular momentum

Authors' addresses: Jingyu Chen, UCLA, Box 951555, Los Angeles, CA, 90095-1555, USA chenjy@g.ucla.edu; Victoria Kala, UCLA, Box 951555, Los Angeles, CA, 90095-1555, USA, vtkala@math.ucla.edu; Alan Marquez-Razon, UCLA, Box 951555, Los Angeles CA, 90095-1555, USA, marqueza04@g.ucla.edu; Elias Gueidon, UCLA, Box 951555, Los Angeles, CA, 90095-1555, USA, egueidon@g.ucla.edu; David A. B. Hyde, UCLA, Box 951555, Los Angeles, CA, 90095-1555, USA, dabh@math.ucla.edu; Joseph Teran, UC Davis, One Shields Ave, Davis, CA, 95616, USA, jteran@math.ucdavis.edu.

Permission to make digital or hard copies of part or all of this work for personal or classroom use is granted without fee provided that copies are not made or distributed for profit or commercial advantage and that copies bear this notice and the full citation on the first page. Copyrights for third-party components of this work must be honored.

For all other uses, contact the owner/author(s).

(c) 2021 Copyright held by the owner/author(s).

0730-0301/2021/8-ART111

https://doi.org/10.1145/3450626.3459874 with Affine-Particle-In-Cell (APIC) [Jiang et al. 2015]. We show that our approach enables implicit time stepping for complex behaviors like the Marangoni effect and hydrophobicity/hydrophilicity. We demonstrate the robustness and utility of our method by simulating materials that exhibit highly diverse degrees of surface tension and thermomechanical effects, such as water, wine and wax.

CCS Concepts: • Mathematics of computing $\rightarrow$ Discretization; Partial differential equations; Solvers; • Applied computing $\rightarrow$ Physics.

Additional Key Words and Phrases: Surface Tension, Momentum Conservation, Melting, Marangoni Effect, Material Point Methods, Particle-In-Cell

\section{ACM Reference Format:}

Jingyu Chen, Victoria Kala, Alan Marquez-Razon, Elias Gueidon, David A. B. Hyde, and Joseph Teran. 2021. A Momentum-Conserving Implicit Material Point Method for Surface Tension with Contact Angles and Spatial Gradients. ACM Trans. Graph. 40, 4, Article 111 (August 2021), 16 pages. https://doi.org/10.1145/3450626.3459874

\section{INTRODUCTION}

Surface tension driven flows like those in milk crowns [Zheng et al. 2015], droplet coalescence [Da et al. 2016; Li et al. 2020; Thürey et al. 2010; Wojtan et al. 2010; Yang et al. 2016a] and bubble formation [Da et al. 2015; Huang et al. 2020; Zhu et al. 2014] comprise some of the most visually compelling fluid motions. Although these effects are most dominant at small scales, increasing demand for realism in computer graphics applications requires modern solvers capable of resolving them. Indeed surface tension effects have been well examined in the computer graphics and broader computational 
physics literature. We design a novel approach for simulating surface tension driven phenomena that arise from spatial variations in cohesion and adhesion forces at the interface between two liquids. This is often called the Marangoni effect [Scriven and Sternling 1960; Venerus and Simavilla 2015] and perhaps the most famous example is the tears of wine phenomenon [Thomson 1855]. Other notable examples of the Marangoni effect include repulsive flows induced by a soap droplet on a water surface as well as the dynamics of molten waxes and metals [Farahi et al. 2004; Langbein 2002].

The spatial variation in the surface forces can be characterized in terms of the potential energy $\Psi^{s}$ associated with surface tension:

$$
\Psi^{s}=\int_{\Gamma} k^{\sigma}(\mathbf{x}) d s(\mathbf{x}) .
$$

Here the surface tension coefficient $k^{\sigma}$ is proportionate to the relative cohesion and adhesion at the interface between the two fluids. Typically this coefficient is constant across the multi-material interface $\Gamma$, however with the Marangoni effect the coefficient varies with $\mathbf{x} \in \Gamma$. These variations are typically driven by temperature or concentration gradients and give rise to many subtle, but important visual behaviors where the variation typically causes fluid to flow away from low surface energy regions towards high surface energy regions. To capture the Marangoni effect, most approaches do not work with the potential energy $\Psi^{s}$ in Equation (1) but instead base their discretization on its first variation. This variation results in the interfacial traction condition

$$
\mathbf{t}=k^{\sigma} \kappa \mathbf{n}+\nabla^{S} k^{\sigma} .
$$

Here $t$ is the force per unit area due to surface tension at the interface $\Gamma, \nabla^{S}$ is the surface gradient operator at the interface and $\kappa$ and $\mathbf{n}$ are the interfacial mean curvature and normal, respectively.

There are many existing techniques in the computational physics literature that resolve spatial variations in the surface tension. For particle-based methods like Smoothed Particle Hydrodynamics (SPH) [Monaghan 1992] and Particle-In-Cell (PIC) [Harlow and Welch 1965], most of these approaches are based on the Continuum Surface Force (CSF) model of Brackbill et al. [1992]. Marangoni effects have not been addressed in computer graphics, other than by Huang et al. [2020] where it was examined for material flows in soap films. The original CSF technique of Brackbill et al. [1992] resolves the mean curvature term in Equation (2), but not the surface gradient term. Tong and Browne [2014] show that the CSF approach can be modified to resolve the surface gradient. However, while this and other existing approaches in the SPH and PIC literature are capable of resolving the spatial variation, none support implicit time stepping for the surface tension forces.

We build on the work of Hyde et al. [2020] and show that efficient implicit time stepping with Marangoni effects is achievable with PIC. As in [Hyde et al. 2020] we observe that similarities with hyperelasticity suggest that the Material Point Method (MPM) [Sulsky et al. 1994] is the appropriate version of PIC. We show that by building our discretization from the energy in Equation (1) rather than the more commonly adopted traction condition in Equation (2), we can naturally compute the first and second variations of the potential needed when setting up and solving the nonlinear systems of equations associated with fully implicit temporal discretization.
Interestingly, by basing our discretization on the energy in Equation (1), we also show that no special treatment is required for the interfacial spatial gradient operator $\nabla^{s}$ as was done in e.g., [Tong and Browne 2014]. Furthermore, we show that our approach to discretizing the Marangoni forces can also be used to impose the contact angle at liquid/solid/air interfaces [Young 1805]. We show that this naturally allows for simulation of droplet streaking effects.

While our method is a generalization of the MPM technique in [Hyde et al. 2020], we also improve on its core functionality. The Hyde et al. [2020] approach is characterized by the introduction of additional surface tension particles at each time step which is designed to represent the liquid interface $\Gamma$ and its area weighted boundary normals. These surface tension particles are temporary and are deleted at the end of the time step to prevent excessive growth in particle count or macroscopic particle resampling. However, the surface tension particles are massless to prevent violation of conservation of particle mass and momentum. We show that this breaks the perfect conservation of grid linear and angular momentum when particles introduce grid nodes with no mass that the surface tension forces will act upon. This is an infrequent occurrence, but breaks the otherwise perfect conservation of grid linear and angular momentum expected with conservative MPM forces. We design a novel mass and momentum resampling technique that, with the introduction of two new types of temporary particles, can restore perfect conservation of grid linear and angular momentum. We call these additional temporary particles balance particles. We show that our novel resampling provides improved behavior over the original approach of Hyde et al. [2020], even in the case of standard, non-Marangoni surface tension effects. Furthermore, although other resampling techniques exist for PIC methods [Edwards and Bridson 2012; Gao et al. 2017b; Yue et al. 2015], we note that ours is the first to guarantee perfect conservation when using generalized particle velocities associated with the Affine Particle-in-Cell (APIC) method [Fu et al. 2017; Jiang et al. 2015, 2016].

Since variations in surface energy are typically based on temperature and/or concentration gradients, we couple our surface tension coefficients with thermodynamically driven quantities. Furthermore, we resolve solid to liquid and liquid to solid phase changes as a function of temperature since many Marangoni effects arise from melting and cooling. Notably, we show that our novel conservative resampling naturally improves discretization of Robin and Neumann boundary conditions on the interface $\Gamma$ needed for convection/diffusion of temperature and concentration. In summary, our primary contributions are:

- A novel implicit MPM discretization of spatially varying surface tension forces.

- A momentum-conserving particle resampling technique for particles near the surface tension liquid interface.

- An implicit MPM discretization of the convection/diffusion evolution of temperature/concentration coupled to the surface tension coefficient including a novel particle-based Robin boundary condition. 


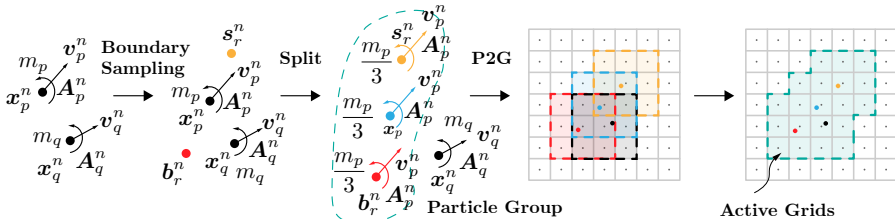

Fig. 2. Splitting. After surface particles (yellow) are created, the mass and momentum of the interior MPM particles (blue) that are closest to the surface particles are immediately distributed. Particles in each particle group are assigned equal mass. MPM particles (black) that are not paired with any surface particles remain intact for the splitting process. Surface particles (yellow) and balance particles (red) are assigned the same linear velocity and affine velocity of the original particle (blue).

\section{RELATED WORK}

We discuss relevant particle-based techniques for simulating Marangoni and surface tension effects, contact angle imposition, thermodynamic evolution of temperature and/or concentration, as well as resampling techniques in particle-based methods.

Particle Methods: Particle-based methods are very effective for computer graphics applications requiring discretization of surface tension forces. Hyde et al. [2020] provide a thorough discussion of the state of the art. Our approach utilizes the particle-based MPM [de Vaucorbeil et al. 2020; Sulsky et al. 1994] PIC technique, largely due to its natural ability to handle self collision [Fei et al. 2018, 2017; Guo et al. 2018; Jiang et al. 2017], topology change [Wang et al. 2019; Wolper et al. 2020, 2019], diverse materials [Daviet and Bertails-Descoubes 2016; Klár et al. 2016; Ram et al. 2015; Schreck and Wojtan 2020; Stomakhin et al. 2013; Wang et al. 2020c; Yue et al. 2015] as well as implicit time stepping with elasticity [Fang et al 2019; Fei et al. 2018; Stomakhin et al. 2013; Wang et al. 2020b]. We additionally use the APIC method [Fu et al. 2017; Jiang et al. 2015, 2016] for its conservation properties and beneficial suppression of noise. Note that our mass and momentum remapping technique is designed to work in the context of the APIC techniques where particles store generalized velocity information.

$\mathrm{SPH}$ is very effective for resolving Marangoni effects. The approaches of Tong and Browne [2014] and Hopp-Hirschler et al. [2018] are indicative of the state of the art. Most SPH works rely on the CSF surface tension model of Brackbill et al. [1992], which transforms surface tension traction into a volumetric force that is only non-zero along (numerically smeared) material interfaces. CSF approaches generally derive surface normal and curvature estimates as gradients of color functions, which can be very sensitive to particle distribution. Also, CSF forces are not exactly conservative [Hyde et al. 2020]. SPH can also be used to simulate the convection and diffusion of temperature/concentration that give rise to the spatial variation in surface energy in the Marangoni effect. Hu and Eberhard [2017] simulate Marangoni convection in a melt pool during laser welding and Russell [2018] does so in laser fusion additive manufacturing processes. Both approaches use SPH with [Tong and Browne

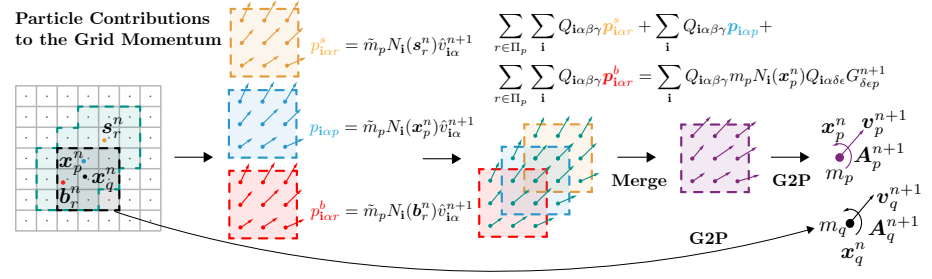

Fig. 3. Merging. The merging process is a modified version of G2P. For the particles that are not associated with surface particles (black), a regular G2P is performed. Among each particle group, we calculate each particle's contribution to the grid momentum and the generalized affine moments of their summed momenta about their center of mass. Then, we restore the mass of the original particle associated with the group prior to the split and compute its generalized affine inertia tensor from its grid mass distribution. Using the affine inertia tensor of the original particle, we compute generalized velocity of the particle after the merging from the generalized moments of the group.

2014] for discretization of Equation (2). Although SPH is very effective for resolving Marangoni effects, all existing approaches utilize explicit treatment of Marangoni forces.

Marangoni effect and contact angle: The Marangoni effect is visually subtle and has not been resolved with particle-based methods in computer graphics applications. Perhaps the most visually compelling example of the Marangoni effect is the tears of wine phenomenon on the walls of a wine glass [Scriven and Sternling 1960; Venerus and Simavilla 2015]. Tears of wine were simulated in [Azencot et al. 2015]. However, the authors modeled the fluid using thin film equations under the lubrication approximation and did not model surface tension gradients. The fingering instabilities they observed are stated to occur due to the asymmetric nature of their initial conditions. The Marangoni effect was resolved by Huang et al. [2020] recently with thin soap films to generate compelling dynamics of the characteristic rainbow patterns in bubbles. Relatedly, Ishida et al. [2020] simulated the evolution of soap films including effects of thin-film turbulence, draining, capillary waves, and evaporation. Outside of computer graphics, the Marangoni effect has been recently studied in works like [Dukler et al. 2020], which models undercompressive shocks in the Marangoni effect, and [de Langavant et al. 2017], which presents a spatially-adaptive level set approach for simulating surfactant-driven flows. Also, Nas and Tryggvason [2003] simulated thermocapillary motion of bubbles and drops in flows with finite Marangoni numbers (flows with significant transport due to Marangoni effects).

Our approach for the Marangoni effect also allows for imposition of contact angles at air/liquid/solid interfaces. This effect is important for visual realism when simulating droplets of water in contact with solid objects like the ground. Contact angles are influenced by the hydrophilicity/hydrophobicity of the surface on which these droplets move or rest [Cassie and Baxter 1944; Johnson Jr. and Dettre 1964]. Wang et al. [2007] solve General Shallow Wave Equations, including surface tension boundary conditions and the virtual surface method of Wang et al. [2005], in order to model contact angles and hydrophilicity. Zheng et al. [2015] extend the virtual fluid surface 
into the solid boundary and apply additional force to achieve the desired contact angles. Yang et al. [2016b] use a pairwise force model [Tartakovsky and Meakin 2005] for handling contact angles in their SPH treatment of fluid-fluid and solid-fluid interfaces. Clausen et al. [2013] also consider the relation between surface tension and contact angles in their Lagrangian finite element approach.

Particle Resampling: Reseeding or resampling particles is a common concern in various simulation methods; generally speaking, particles need to be distributed with sufficient density near dynamic areas of flow or deformation in order to accurately resolve the dynamics of the system [Ando et al. 2012; Losasso et al. 2008; Narain et al. 2010]. Edwards and Bridson [2012] use a non-conservative random sampling scheme to seed and reseed particles with PIC. Pauly et al. [2005] resample to preserve detail near cracks/fracture, but their resampling does not attempt to conserve momentum. Yue et al. [2015] use Poisson disk sampling to insert new points in lowdensity regions and merge points that are too close to one another with MPM. However, their resampling method is not demonstrated to be momentum-conserving. A conservative variant of this splitand-merge approach is applied in [Gao et al. 2017b] where mass and linear momentum are conserved during particle splitting and merging. However, angular momentum conservation is not conserved. Furthermore, these techniques use PIC, not APIC, and neither is designed to guarantee conservation with the generalized velocity state in APIC techniques [Fu et al. 2017; Jiang et al. 2015, 2016].

Thermomechanical Effects: Thermodynamic effects in visual simulation date back to at least Terzopoulos et al. [1991]. More recently, melting and resolidification for objects like melting candles have been simulated using various methods, including Lattice Boltzmann [Wang et al. 2012] and SPH [Lenaerts and Dutré 2009; Paiva et al. 2009], though these results leave room for improved visual and physical fidelity. FLIP methods have also been used for thermodynamic problems, such as [Gao et al. 2017a], which adapts the latent heat model from [Stomakhin et al. 2014]. Condensation and evaporation of water were considered in several works based on SPH [Hochstetter and Kolb 2017; Zhang et al. 2017]. SPH was also applied to the problem of simulating boiling bubbles in [Gu and Yang 2016], which models heat conduction, convection and mass transfer. Recently, particle-based thermodynamics models were incorporated into an SPH snow solver [Gissler et al. 2020a], with temperature-dependent material properties such as the Young's modulus. In another vein, Pirk et al. [2017] used position-based dynamics and Cosserat physics to simulate combustion of tree branches, including models for moisture and charring. Yang et al. [2017] used the Cahn-Hilliard and Allen-Cahn equations to evolve a continuous phase variable for materials treated with their phase-field method. Maeshima et al [2020] considered particle-scale explicit MPM modeling for additive manufacturing (selective laser sintering) that included a latent heat model for phase transition. For a detailed review of thermodynamical effects in graphics, we refer the reader to [Stomakhin et al. 2014].

Surface Tension: Many methods for simulating non-Marangoni surface tension effects have been developed for computer graphics applications. We refer the reader to [Hyde et al. 2020] for a detailed survey. More recently, Chen et al. [2020] incorporated subcell-accurate surface tension forces in an Eulerian fluid framework based on integrating the mean curvature flow of the liquid interface (following Sussman and Ohta [2009]). With an eye towards resolving codimensional flow features, such as thin sheets and filaments, Batty et al. [2012] utilized an energy based approach for surface tension. Wang et al. [2020a] and Zhu et al. [2014] also simulated surface tension forces with codimensional features using movingleast-squares particles and simplicial complexes, respectively. Most related to the present work, Hyde et al. [2020] proposed an implicit material point method for simulating liquids with large surface energy, such as liquid metals. Their surface tension formulation follows [Adamson and Gast 1967; Brackbill et al. 1992; Buscaglia and Ausas 2011] and incorporates a potential energy associated with surface tension into the MPM framework. Material boundaries are sampled using massless MPM particles.

\section{GOVERNING EQUATIONS}

We first define the governing equations for thermomechanically driven phase change of hyperelastic solids and liquids with variable surface energy. As in [Hyde et al. 2020] we also cover the updated Lagrangian kinematics. Lastly, we provide the variational form of the governing equations for use in MPM discretization. We note that throughout the document Greek subscripts are assumed to run from $0,1, \ldots, d-1$ for the dimension $d=2,3$ of the problem. Repeated Greek subscripts imply summation, while sums are explicitly indicated for Latin subscripts. Also, Latin subscripts in bold are used for multi-indices.

\subsection{Kinematics}

We adopt the continuum assumption [Gonzalez and Stuart 2008] and updated Lagrangian kinematics [Belytschko et al. 2013] used by Hyde et al. [2020]. At time $t$ we associate our material with subsets $\Omega^{t} \subset \mathbb{R}^{d}, d=2,3$. We use $\Omega^{0}$ to denote the initial configuration of material with $\mathrm{X} \in \Omega^{0}$ used to denote particles of material at time $t=0$. A flow map $\phi: \Omega^{0} \times[0, T] \rightarrow \mathbb{R}^{d}$ defines the material motion of particles $\mathrm{X} \in \Omega^{0}$ to their time $t$ locations $\mathbf{x} \in \Omega^{t}$ as $\phi(X, t)=\mathbf{x}$. The Lagrangian velocity is defined by differentiating the flow map in time $\mathbf{V}(\mathbf{X}, t)=\frac{\partial \phi}{\partial t}(\mathbf{X}, t)$.

3.1.1 Eulerian and Updated Lagrangian Representations. The Lagrangian velocity can be difficult to work with in practice since real world observations of material are made in $\Omega^{t}$ not $\Omega^{0}$. The Eulerian velocity $\mathbf{v}: \Omega^{t} \rightarrow \mathbb{R}^{d}$ is what we observe in practice. The Eulerian velocity is defined in terms of the inverse flow map $\phi^{-1}(\cdot, t): \Omega^{t} \rightarrow \Omega^{0}$ as $\mathbf{v}(\mathbf{x}, t)=\mathbf{V}\left(\phi^{-1}(\mathbf{x}, t), t\right)$ where $\phi^{-1}(\mathbf{x}, t)=$ $\mathrm{X}$. In general, we can use the flow map and its inverse to pullback quantities defined over $\Omega^{t}$ and pushforward quantities defined over $\Omega^{0}$, respectively. For example, given $G: \Omega^{0} \rightarrow \mathbb{R}$, its pushforward $g: \Omega^{t} \rightarrow \mathbb{R}$ is defined as $g(\mathbf{x})=G\left(\phi^{-1}(\mathbf{x}, t)\right)$. This process is related to the material derivative operator $\frac{D}{D t}$ where $\frac{D g}{D t}(\mathbf{x}, t)=\frac{\partial G}{\partial t}\left(\phi^{-1}(\mathbf{x}, t)\right)=\frac{\partial g}{\partial t}(\mathbf{x}, t)+\sum_{\alpha=0}^{d-1} \frac{\partial g}{\partial x_{\alpha}}(\mathbf{x}, t) v_{\alpha}(\mathbf{x}, t)$ (see e.g., [Gonzalez and Stuart 2008] for more detail).

In the updated Lagrangian formalism [Belytschko et al. 2013] we write quantities over an intermediate configuration of material $\Omega^{s}$ 
with $0 \leq s<t$. For example, we can define $\hat{g}(\cdot, s): \Omega^{s} \rightarrow \mathbb{R}$ as $\hat{g}(\tilde{\mathbf{x}}, s)=G\left(\phi^{-1}(\tilde{\mathbf{x}}, s)\right)$ for $\tilde{\mathbf{x}} \in \Omega^{s}$. As shown in [Hyde et al. 2020], this is particularly useful when discretizing momentum balance using its variational form. The key observation is that the updated Lagrangian velocity can be written as $\hat{\mathbf{v}}(\tilde{\mathbf{x}}, s, t)=\mathbf{V}\left(\phi^{-1}(\tilde{\mathbf{x}}, s), t\right)=$ $\mathbf{v}(\hat{\boldsymbol{\phi}}(\tilde{\mathbf{x}}, s, t), t)$ with $\hat{\boldsymbol{\phi}}(\tilde{\mathbf{x}}, s, t)=\boldsymbol{\phi}\left(\boldsymbol{\phi}^{-1}(\tilde{\mathbf{x}}, s), t\right)$ for $\tilde{\mathbf{x}} \in \Omega^{s}$. Intuitively, $\hat{\boldsymbol{\phi}}(\cdot, s, t): \Omega^{s} \rightarrow \Omega^{t}$ is the mapping from the time $s$ configuration to the time $t$ configuration induced by the flow map. This has a simple relation to the material derivative as $\frac{\partial \hat{\mathbf{V}}}{\partial t}(\tilde{\mathbf{x}}, s, t)=\frac{\partial \mathbf{V}}{\partial t}\left(\phi^{-1}(\tilde{\mathbf{x}}, s), t\right)=$ $\frac{D \mathbf{v}}{D t}(\hat{\boldsymbol{\phi}}(\tilde{\mathbf{x}}, s, t), t)$. As in [Hyde et al. 2020] we will generally use upper case for Lagrangian quantities, lower case for Eulerian quantities and hat superscripts for updated Lagrangian quantities.

3.1.2 Deformation Gradient. The deformation gradient $\mathrm{F}=\frac{\partial \phi}{\partial \mathrm{X}}$ is defined by differentiating the flow map in space and can be used to quantify the amount of deformation local to a material point. We use $J=\operatorname{det}(\mathbf{F})$ to denote the deformation gradient determinant. $J$ represents the amount of volumetric dilation at a material point. Furthermore, it is used when changing variables with integration. We also make use of similar notation for the $\hat{\boldsymbol{\phi}}$ mapping from $\Omega^{s}$ to $\Omega^{t}$, i.e. $\hat{\mathbf{F}}=\frac{\partial \hat{\phi}}{\partial \tilde{\mathbf{x}}}, \hat{J}=\operatorname{det}(\hat{\mathbf{F}})$.

\subsection{Conservation of Mass and Momentum}

Our governing equations primarily consist of conservation of mass and momentum which can be expressed as

$$
\rho \frac{D \mathbf{v}}{D t}=\nabla \cdot \boldsymbol{\sigma}+\rho \mathbf{g}, \frac{D \rho}{D t}=-\rho \nabla \cdot \mathbf{v}, \mathbf{x} \in \Omega^{t}
$$

where $\rho$ is the Eulerian mass density, $\mathbf{v}$ is the Eulerian material velocity, $\sigma$ is the Cauchy stress and $\mathrm{g}$ is gravitational acceleration. Boundary conditions for these equations are associated with a free surface for solid material, surface tension for liquids and/or prescribed velocity conditions. We use $\partial \Omega_{N}^{t}$ to denote the portion of the time $t$ boundary subject to free surface or surface tension conditions and $\partial \Omega_{D}^{t}$ to denote the portion of the boundary with Dirichlet velocity boundary conditions. Free surface conditions and surface tension boundary conditions are expressed as

$$
\boldsymbol{\sigma} \mathbf{n}=\mathbf{t}, \mathbf{x} \in \partial \Omega_{N}^{t}
$$

where $\mathbf{t}=\mathbf{0}$ for free surface conditions and $\mathbf{t}=k^{\sigma} \kappa \mathbf{n}+\nabla^{S} k^{\sigma}$ from Equation (2) for surface tension conditions. Velocity boundary conditions may be written as

$$
\mathbf{v} \cdot \mathbf{n}=v_{\mathrm{bc}}^{n}, \mathbf{x} \in \partial \Omega_{D}^{t} .
$$

3.2.1 Constitutive Models. Each material point is either a solid or liquid depending on the thermomechanical evolution. For liquids, the Cauchy stress $\sigma$ is defined in terms of pressure and viscous stress:

$$
\boldsymbol{\sigma}=-p \mathbf{I}+\mu\left(\frac{\partial \mathbf{v}}{\partial \mathbf{x}}+\frac{\partial \mathbf{v}^{T}}{\partial \mathbf{x}}\right), p=-\frac{\partial \psi^{p}}{\partial J},
$$

with $\psi^{p}(J)=\frac{\lambda^{l}}{2}(J-1)^{2}$. Here $\lambda^{l}$ is the bulk modulus of the liquid and $\mu^{l}$ is its viscosity. For solids, the Cauchy stress is defined in terms of a hyperelastic potential energy density $\psi^{s}$ as

$$
\boldsymbol{\sigma}=\frac{1}{j} \frac{\partial \psi^{s}}{\partial \mathbf{F}} \mathbf{f}^{T}
$$

where $\mathbf{f}(\mathbf{x}, t)=\mathbf{F}\left(\boldsymbol{\phi}^{-1}(\mathbf{x}, t), t\right)$ and $j(\mathbf{x}, t)=J\left(\phi^{-1}(\mathbf{x}, t), t\right)$ are the Eulerian deformation gradient and its determinant, respectively. We use the fixed-corotated constitutive model from [Stomakhin et al. 2012] for $\psi^{h}$. This model is defined in terms of the polar SVD [Irving et al. 2004] of the deformation gradient $\mathbf{F}=\mathbf{U} \Sigma \mathbf{V}^{T}$ with

$$
\psi^{h}(\mathbf{F})=\mu^{h} \sum_{\alpha=0}^{d-1}\left(\sigma_{\alpha}-1\right)^{2}+\frac{\lambda^{h}}{2}(J-1)^{2},
$$

where the $\sigma_{\alpha}$ are the diagonal entries of $\Sigma$ and $\mu^{h}, \lambda^{h}$ are the hyperelastic Lamé coefficients.

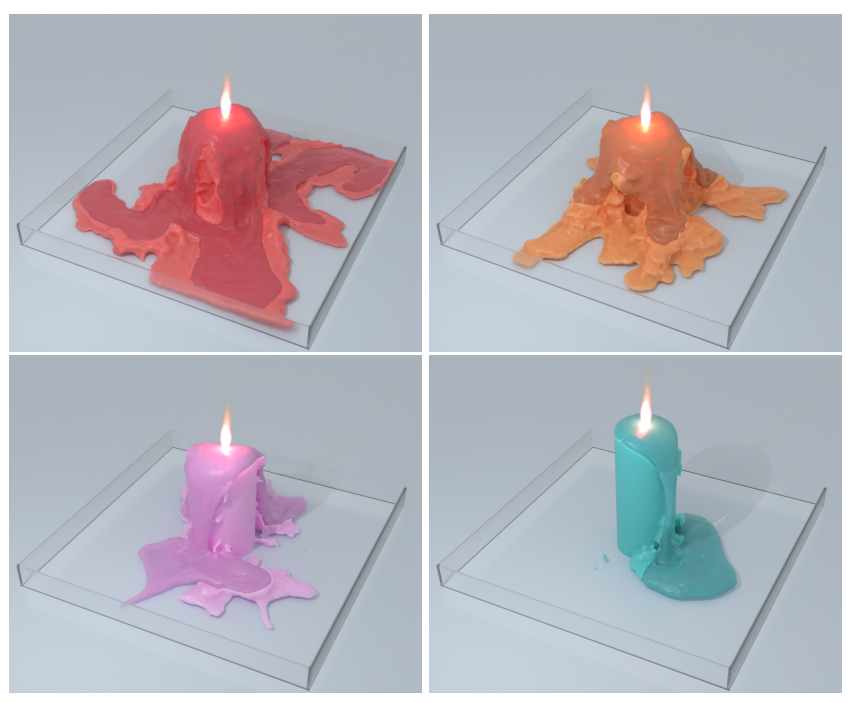

Fig. 4. Various $k^{\sigma}$ values $(0.05,0.1,0.2,0.4 \mathrm{~N} / \mathrm{m})$ are simulated in the case of a melting candle. Frame 1202 is shown.

\subsection{Conservation of energy}

We assume the internal energy of our materials consists of potential energy associated with surface tension, liquid pressure and hyperelasticity and thermal energy associated with material temperature. Conservation of energy together with thermodynamic considerations requires convection/diffusion of the material temperature [Gonzalez and Stuart 2008] subject to Robin boundary conditions associated with convective heating by ambient material:

$$
\begin{aligned}
\rho c_{p} \frac{D T}{D t} & =K \nabla^{2} T+H \\
K \nabla T \cdot \mathbf{n} & =-h(T-\bar{T})+b .
\end{aligned}
$$

Here $c_{p}$ is specific heat capacity, $T$ is temperature, $K$ is thermal conductivity, $H$ is a source function, $\bar{T}$ is the temperature of ambient material, and $\mathbf{n}$ is the surface boundary normal. $h$ controls the rate of convective heating to the ambient temperature, and $b$ represents the rate of boundary heating independent of the ambient material temperature $\bar{T}$.

The total potential energy $\Psi$ in our material is as in [Hyde et al. 2020], however we include the spatial variation of the surface energy density in Equation (1) and the hyperelastic potential for solid 
regions:

$$
\Psi(\phi(\cdot, t))=\Psi^{\sigma}(\phi(\cdot, t))+\Psi^{l}(\phi(\cdot, t))+\Psi^{h}(\phi(\cdot, t))+\Psi^{g}(\phi(\cdot, t)) .
$$

Here $\Psi^{\sigma}$ is the potential from surface tension, $\Psi^{l}$ is the potential from liquid pressure, $\Psi^{h}$ is the potential from solid hyperelasticity, and $\Psi^{g}$ is the potential from gravity. As in typical MPM discretizations, our approach is designed in terms of these energies:

$$
\begin{array}{ll}
\Psi^{\sigma}(\boldsymbol{\phi}(\cdot, t))=\int_{\partial \Omega^{t}} k^{\sigma}(\mathbf{x}, t) d s(\mathbf{x}), & \Psi^{g}\left(\boldsymbol{\phi}(\cdot, t)=\int_{\Omega^{0}} R \mathbf{g} \cdot \boldsymbol{\phi} J d \mathbf{X},\right. \\
\Psi^{l}(\phi(\cdot, t))=\int_{\Omega^{0}} \frac{\lambda^{l}}{2}(J-1)^{2} d \mathbf{X}, & \Psi^{h}(\phi(\cdot, t))=\int_{\Omega^{0}} \psi^{h}(\mathbf{F}) d \mathbf{X} .
\end{array}
$$

Here $R$ is the pullback (see Section 3.1) of the mass density $\rho$. Note that in the expression for the surface tension potential it is useful to change variables using the updated Lagrangian view as in [Hyde et al. 2020]:

$\Psi^{\sigma}(\boldsymbol{\phi}(\cdot, t))=\int_{\partial \Omega^{t}} k^{\sigma}(\mathbf{x}, t) d s(\mathbf{x})=\int_{\partial \Omega^{s}} k^{\sigma}(\hat{\boldsymbol{\phi}}(\tilde{\mathbf{x}}, s, t), t)\left|\hat{J} \hat{\mathbf{F}}^{-T} \tilde{\mathbf{n}}\right| d s(\tilde{\mathbf{x}})$.

Here $\tilde{\mathbf{n}}$ is the outward unit normal at a point on the boundary of $\Omega^{s}$ and the expression $d s(\mathbf{x})=\left|\hat{J}^{-T} \tilde{\mathbf{F}}\right| d s(\tilde{\mathbf{x}})$ arises by a change of variables from an integral over $\partial \Omega^{t}$ to one over $\partial \Omega^{s}$. Notably, the spatial variation in $k^{\sigma}$ is a natural extension of the Hyde et al. [2020] approach.

\subsection{Variational Form of Momentum Balance}

The strong form of momentum balance in Equation (3), together with the traction (Equation (4)) and Dirichlet velocity boundary conditions (Equation (5)), is equivalent to a variational form that is useful when discretizing our governing equations using MPM. To derive the variational form, we take the dot product of Equation (3) with an arbitrary function $\mathbf{w}: \Omega^{t} \rightarrow \mathbb{R}^{d}$ satisfying $\mathbf{w} \cdot \mathbf{n}=0$ for $\mathbf{x} \in \partial \Omega_{D}^{t}$ and integrate over the domain $\Omega^{t}$, applying integration by parts where appropriate. Requiring that the Dirichlet velocity conditions in Equation (4) hold together with the following integral equations for all functions $\mathrm{w}$ is equivalent to the strong form, assuming sufficient solution regularity:

$$
\int_{\Omega^{t}} \rho \frac{D v_{\alpha}}{D t} w_{\alpha} d \mathbf{x}=-\frac{d}{d \epsilon} P E(0 ; \mathbf{w})-\mu^{l} \int_{\Omega^{t}} \epsilon_{\alpha \beta}^{v} \epsilon_{\alpha \beta}^{w} d \mathbf{x} .
$$

Here $\epsilon^{w}=\frac{1}{2}\left(\frac{\partial w_{\alpha}}{\partial x_{\beta}}+\frac{\partial w_{\beta}}{\partial x_{\alpha}}\right)$ and $\epsilon^{v}=\frac{1}{2}\left(\frac{\partial v_{\alpha}}{\partial x_{\beta}}+\frac{\partial v_{\beta}}{\partial x_{\alpha}}\right)$ and

$$
\operatorname{PE}(\epsilon ; \mathbf{w})=\Psi(\phi(\cdot, t)+\epsilon \mathbf{W}) .
$$

Here $\mathbf{W}$ is the pullback of $\mathbf{w}$ (see Section 3.1). Note that this notation is rather subtle for the surface tension potential energy. For clarification,

$$
\Psi^{\sigma}(\boldsymbol{\phi}(\cdot, t)+\epsilon \mathbf{W})=\int_{\partial \Omega^{s}} k^{\sigma}(\hat{\boldsymbol{\phi}}+\epsilon \hat{\mathbf{w}})\left|\hat{J}_{\epsilon, \hat{\mathbf{w}}} \hat{\mathbf{F}}_{\epsilon, \hat{\mathbf{w}}}^{-T} \tilde{\mathbf{n}}\right| d s(\tilde{\mathbf{x}}),
$$

where $\hat{\mathbf{w}}(\tilde{\mathbf{x}}, s, t)=\mathbf{w}(\hat{\boldsymbol{\phi}}(\tilde{\mathbf{x}}, s, t))$ is the updated Lagrangian pullback of $\mathbf{W}, \hat{\mathbf{F}}_{\epsilon, \hat{\mathbf{w}}}=\frac{\partial \hat{\boldsymbol{\phi}}+\epsilon \hat{\mathbf{W}}}{\partial \tilde{\mathbf{x}}}$ is the deformation gradient of the mapping $\hat{\boldsymbol{\phi}}+$ $\epsilon \hat{\mathbf{w}}$ and $\hat{J}_{\epsilon, \hat{\mathbf{w}}}=\operatorname{det}\left(\hat{\mathbf{F}}_{\epsilon, \hat{\mathbf{w}}}\right)$ is its determinant. Lastly, for discretization purposes, in practice we change variables in the viscosity term

$$
\mu^{l} \int_{\Omega^{t}} \epsilon_{\alpha \beta}^{v} \epsilon_{\alpha \beta}^{w} d \mathbf{x}=\mu^{l} \int_{\Omega^{s}} \hat{\epsilon}_{\alpha \beta}^{v} \hat{\epsilon}_{\alpha \beta}^{w} \hat{J} d \tilde{\mathbf{x}}
$$

We can similarly derive a variational form of the temperature evolution in Equation (6) by requiring

$$
\begin{aligned}
\int_{\Omega^{t}} \rho c_{p} \frac{D T}{D t} q d \mathbf{x}= & -\int_{\partial \Omega^{t}} q h T d \mathbf{S}(\mathbf{x})+\int_{\partial \Omega^{t}} q(h \bar{T}+b) d \mathbf{S}(\mathbf{x}) \\
& +\int_{\Omega^{t}} H q d \mathbf{x}-\int_{\Omega^{t}} \nabla q \cdot K \nabla T d \mathbf{x}
\end{aligned}
$$

for all functions $q: \Omega^{t} \rightarrow \mathbb{R}$.

\subsection{Thermomechanical Material Dependence and Phase Change}

The thermomechanical material dependence is modeled by allowing the surface tension coefficient $k^{\sigma}$, the liquid bulk modulus $\lambda^{l}$, the liquid viscosity $\mu^{l}$ and the hyperelastic Lamé coefficients $\mu^{h}, \lambda^{h}$ to vary with temperature. When $T$ exceeds a user-specified melting point $T_{\text {melt }}$, the solid phase is changed to liquid and the deformation gradient determinant $J$ is set to 1 . Similarly, if the liquid temperature drops below $T_{\text {melt }}$, the phase is updated to be hyperelastic solid, and the deformation gradient $\mathbf{F}$ is set to the identity matrix. In practice, resetting the deformation gradient and its determinant helps to prevent nonphysical popping when the material changes from solid to liquid and vice versa. We remark that incorporating a more sophisticated phase change model, such as a latent heat buffer, is potentially useful in future work [Stomakhin et al. 2014].

\subsection{Contact Angle}

The contact angle between a liquid, a solid boundary and the ambient air is governed by the Young equation [Young 1805]. This expression relates the resting angle $\theta$ (measured through the liquid) of a liquid in contact with a solid surface to the surface tension coefficients between the liquid, solid and air phases:

$$
k_{S G}^{\sigma}=k_{S L}^{\sigma}+k_{L G}^{\sigma} \cos (\theta) \text {. }
$$

The surface tension coefficients are between the solid and gas phases, solid and liquid phases, and liquid and gas phases, respectively. As in [Clausen et al. 2013], we assume $k_{S G}^{\sigma}$ is negligible since we are using a free surface assumption and do not explicitly model the air. Under this assumption, the solid-liquid contact angle is determined by the surface tension ratio $-k_{S L}^{\sigma} / k_{L G}^{\sigma}$. We note that, while one would expect surface tension coefficients/energies to be positive, this ratio can be negative under the assumption of zero solid-gas surface tension. Furthermore, we note that utilizing this expression requires piecewise constant surface tension coefficients where the variation along the liquid boundary is based on which portion is in contact with the air and which is in contact with the solid. The distinct surface tension coefficients on different interfaces provide controllability of the spreading behavior of the liquid on the solid surface.

\section{DISCRETIZATION}

As in [Hyde et al. 2020], we use MPM [Sulsky et al. 1994] and APIC [Jiang et al. 2015] to discretize the governing equations. The domain $\Omega^{t^{n}}$ at time $t^{n}$ is sampled using material points $\mathrm{x}_{p}^{n}$. These points also store approximations of the deformation gradient determinant $J_{p}^{n}$, constant velocity $\mathbf{v}_{p}^{n}$, affine velocity $\mathbf{A}_{p}^{n}$, volume $V_{p}^{0}$, mass $m_{p}=$ 
$\rho\left(\mathbf{x}_{p}^{0}, t^{0}\right) V_{p}^{0}$, temperature $T_{p}^{n}$, and temperature gradient $\nabla T_{p}^{n}$. We also make use of a uniform background grid with spacing $\Delta x$ when discretizing momentum updates. To advance our state to time $t^{n+1}$, we use the following steps:

(1) Resample particle boundary for surface tension and Robin boundary temperature conditions.

(2) P2G: Conservative transfer of momentum and temperature from particles to grid.

(3) Update of grid momentum and temperature.

(4) G2P: Conservative transfer of momentum and temperature from grid to particles.

\subsection{Conservative Surface Particle Resampling}

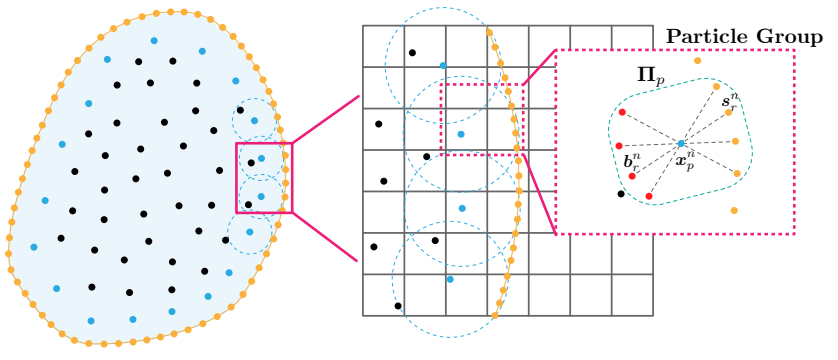

Fig. 5. A portion of an MPM fluid in the simulation domain. Surface particles (yellow) are sampled on faces of the zero isocontour of the level set formed by unioning spherical level sets around each MPM particle. Each surface particle generates an associated balance particle (red) such that the closest MPM particle (blue) to a boundary particle lies on the midpoint of a line segment between the surface particle and balance particle. A single blue particle at $\boldsymbol{x}_{p}$ may be paired with multiple surface particles and balance particles, and they are considered to be in a particle group $\Pi_{p}$. MPM particles that are not associated with any surface tension particles are marked as black.

The integrals associated with the surface tension energy in Equation (7) and the Robin temperature condition in Equation (10) are done over the boundary of the domain. We follow Hyde et al. [2020] and introduce special particles to cover the boundary in order to serve as quadrature points for these integrals. As in [Hyde et al 2020] these particles are temporary and are removed at the end of the time step. However, while Hyde et al. [2020] used massless surface particles, we design a novel conservative mass and momentum resampling for surface particles. Massless particles easily allow for momentum conserving transfers from particles to the grid and vice versa; however, they can lead to loss of conservation in the grid momentum update step. This occurs when there is a grid cell containing only massless particles. In this case, there are grid nodes with no mass that receive surface tension forces. These force components are then effectively thrown out since only grid nodes with mass will affect the end of time step particle momentum state (see Section 6.1).

We resolve this issue by assigning mass to each of the surface particles. However, to conserve total mass, some mass must be subtracted from interior MPM particles. Furthermore, changing the mass of existing particles also changes their momentum, which may lead to violation of conservation. In order to conserve mass, linear momentum and angular momentum, we introduce a new particle for each surface particle. We call these balance particles, and like surface particles they are temporary and will be removed at the end of the time step. We show that the introduction of these balance particles naturally allows for conservation both when they are created at the beginning of the time step and when they are removed at the end of the time step.

4.1.1 Surface Particle Sampling. We first introduce surface particles using the approach in [Hyde et al. 2020]. A level set enclosing the interior MPM particles is defined as the union of spherical level sets defined around each interior MPM particle. Unlike Hyde et al. [2020], we do not smooth or shift the unioned level set. We compute the zero isocontour of the level set using marching cubes [Chernyaev 1995] and randomly sample surface particles along this explicit representation. In [Hyde et al. 2020], three-dimensional boundaries were sampled using a number of sample points proportional to the surface area of each triangle. Sample points were computed using uniform random barycentric weights, which leads to a nonuniform distribution of points in each triangle. Instead, we employ a strategy of per-triangle Monte Carlo sampling using a robust Poisson distribution, as described in Figure 1 of [Corsini et al. 2012] (not their blue noise algorithm); uniform triangle sample points are generated as in [Osada et al. 2002]. We found that this gave better coverage of the boundary without generating particles in a biased, mesh-dependent fashion (see Figure 6). We note that radii for the particle level sets are taken to be $0.73 \Delta x$ (slightly larger than $\frac{\sqrt{2}}{2} \Delta x$ ) in $2 \mathrm{D}$ and $0.867 \Delta x$ (slightly larger than $\frac{\sqrt{3}}{2} \Delta x$ ) in $3 \mathrm{D}$, where $\Delta x$ is the MPM grid spacing. This guarantees that even a single particle in isolation will always generate a level set zero isocontour that intersects the grid and will therefore always generate boundary sample points. Note also that as in [Hyde et al. 2020], we use the explicit marching cubes mesh of the zero isocontour to easily and accurately generate samples of area weighted normals $d \mathrm{~A}_{r}^{n}$ where $\sum\left|d \mathbf{A}_{r}^{n}\right| \approx \int_{\partial \Omega^{t^{n}}} d \mathbf{s}$ are chosen with direction from the triangle normal and magnitude based on the number of samples in a given triangle and the triangle area.

4.1.2 Balance Particle Sampling. For each surface particle $\mathrm{s}_{r}^{n}$, we additionally generate a balance particle $\mathbf{b}_{r}^{n}$. First, we compute the closest interior MPM particle for each surface particle $\mathbf{x}_{p\left(s_{r}^{n}\right)}^{n}$. Then we introduce the corresponding balance particle as

$$
\mathbf{b}_{r}^{n}=\mathbf{s}_{r}^{n}+2\left(\mathbf{x}_{p\left(\mathbf{s}_{r}^{n}\right)}^{n}-\mathbf{s}_{r}^{n}\right) .
$$

4.1.3 Mass and Momentum Splitting. After introducing the surface $\mathbf{s}_{r}^{n}$ and balance $\mathbf{b}_{r}^{n}$ particles, we assign them mass and momentum (see Figure 2). To achieve this in a conservative manner, we first partition the surface particles into particle groups $\Pi_{p}$ defined as the set of surface particle indices $r$ such that $\mathrm{x}_{p}^{n}$ is the closest interior MPM particle to $\mathbf{s}_{r}^{n}$ (see Figure 5). We assign the mass $m_{p}$ of the particle $\mathrm{x}_{p}^{n}$ to the collection of $\mathrm{x}_{p}^{n}, \mathbf{s}_{r}^{n}$ and $\mathbf{b}_{r}^{n}$ for $r \in \Pi_{p}$ uniformly by defining a mass of $\tilde{m}_{p}=\frac{m_{p}}{2\left|\Pi_{p}\right|+1}$ to each surface and balance point as well as to $x_{p}^{n}$. Here $\left|\Pi_{p}\right|$ is the number of elements in the set. This operation is effectively a split of the original particle $\mathbf{x}_{p}^{n}$ with 


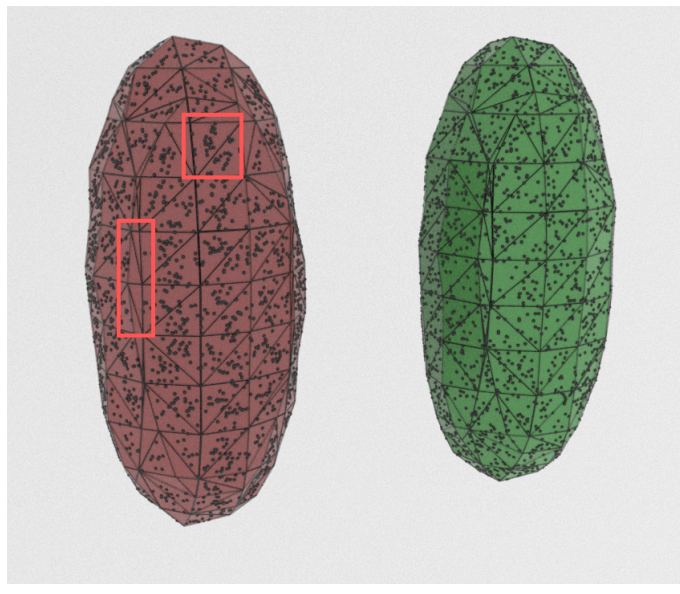

Fig. 6. Isocontour and sampled boundary particles for an ellipsoid. (Left) Using the method of Hyde et al. [2020]. Note how low-quality triangles are undersampled and how sample points often clump near triangle centers. (Right) The present method, which does not suffer from similar issues.

mass $m_{p}$ into a new collection of particles $\mathbf{x}_{p}^{n}, \mathbf{s}_{r}^{n}, \mathbf{b}_{r}^{n}, r \in \Pi_{p}$ with masses $\tilde{m}_{p}$. This split trivially conserves the mass. Importantly, by construction of the balance particles (Equation (12)) we ensure that the center of mass of the collection is equal to the original particle $\mathbf{x}_{p}^{n}$ :

$$
\frac{1}{m_{p}}\left(\tilde{m}_{p} \mathbf{x}_{p}^{n}+\sum_{r \in \Pi_{p}} \tilde{m}_{p} \mathbf{s}_{r}^{n}+\tilde{m}_{p} \mathbf{b}_{r}^{n}\right)=\mathbf{x}_{p}^{n} .
$$

With this particle distribution, conservation of linear and angular momentum can be achieved by simply assigning each new particle in the collection the velocity $\mathbf{v}_{p}^{n}$ and affine velocity $\mathrm{A}_{p}^{n}$ of the original particle $\mathrm{x}_{p}^{n}$. We note that the conservation of the center of mass (Equation (13)) is essential for this simple constant velocity split to conserve linear and angular momentum (see [Chen et al. 2021]).

\subsection{Transfer: P2G}

After the addition of the surface and balance particles, we transfer mass and momentum to the grid in the standard APIC [Jiang et al. 2015] way using their conservatively remapped mass and velocity state

$$
\begin{aligned}
m_{\mathbf{i}}^{n} & =\sum_{p} \tilde{m}_{p}\left(N_{\mathbf{i}}\left(\mathbf{x}_{p}^{n}\right)+\sum_{r \in \Pi_{p}} N_{\mathbf{i}}\left(\mathbf{s}_{r}^{n}\right)+N_{\mathbf{i}}\left(\mathbf{b}_{r}^{n}\right)\right), \\
m_{\mathbf{i}}^{n} \mathbf{v}_{\mathbf{i}}^{n} & =\sum_{p} \tilde{m}_{p} N_{\mathbf{i}}\left(\mathbf{x}_{p}^{n}\right)\left(\mathbf{v}_{p}^{n}+\mathbf{A}_{p}^{n}\left(\mathbf{x}_{\mathbf{i}}-\mathbf{x}_{p}^{n}\right)\right) \\
& +\sum_{p} \tilde{m}_{p} \sum_{r \in \Pi_{p}} N_{\mathbf{i}}\left(\mathbf{s}_{r}^{n}\right)\left(\mathbf{v}_{p}^{n}+\mathrm{A}_{p}^{n}\left(\mathbf{x}_{\mathbf{i}}-\mathbf{s}_{r}^{n}\right)\right) \\
& +\sum_{p} \tilde{m}_{p} \sum_{r \in \Pi_{p}} N_{\mathbf{i}}\left(\mathbf{b}_{r}^{n}\right)\left(\mathbf{v}_{p}^{n}+\mathbf{A}_{p}^{n}\left(\mathbf{x}_{\mathbf{i}}-\mathbf{b}_{r}^{n}\right)\right) .
\end{aligned}
$$

Here $\mathbf{N}_{\mathbf{i}}(\mathbf{x})=\mathbf{N}\left(\mathbf{x}-\mathbf{x}_{\mathbf{i}}\right)$ are quadratic B-splines defined over the uniform grid with $\mathbf{x}_{\mathbf{i}}$ living at cell centers [Stomakhin et al. 2013].
Note that for interior MPM particles far enough from the boundary that $\Pi_{p}=\varnothing$. This reduces to the standard APIC [Jiang et al. 2015] splat since $\tilde{m}_{p}=m_{p}^{n}$. We also transfer temperature from particles to grid using

$$
T_{\mathrm{i} \alpha}^{n}=\sum_{p} m_{p} N_{\mathbf{i}}\left(\mathbf{x}_{p}^{n}\right)\left(T_{p}^{n}+\left(x_{\mathbf{i} \alpha}-x_{p \alpha}^{n}\right) \nabla T_{p \alpha}^{n}\right) .
$$

Note that for the temperature transfer, we only use surface particles to properly apply the thermal boundary conditions, and we do not use these particles to transfer mass-weighted temperature to the grid.

\subsection{Grid Momentum and Temperature Update}

We discretize the governing equations in the standard MPM manner by using the particles as quadrature points in the variational forms. The interior MPM particles $\mathbf{x}_{p}^{n}$ are used for volume integrals and the surface particles $\mathbf{s}_{r}^{n}$ are used for surface integrals. By choosing $s=t^{n}, t=t^{n+1}$ and by using grid discretized versions of $\hat{\mathbf{w}}(\tilde{\mathbf{x}})=$ $\sum_{\mathbf{j}} \mathbf{w}_{\mathbf{j}} N_{\mathbf{j}}(\tilde{\mathbf{x}}), \hat{\mathbf{v}}\left(\tilde{\mathbf{x}}, t^{n}, t^{n+1}\right)=\sum_{\mathbf{i}} \hat{\mathbf{v}}_{\mathbf{i}}^{n+1} N_{\mathbf{i}}(\tilde{\mathbf{x}}), \hat{q}(\tilde{\mathbf{x}})=\sum_{\mathbf{j}} q_{\mathbf{j}} N_{\mathbf{j}}(\tilde{\mathbf{x}})$ and $\hat{T}(\tilde{\mathbf{x}})=\sum_{\mathbf{i}} \hat{T}_{\mathbf{i}} N_{\mathbf{i}}(\tilde{\mathbf{x}})$.

4.3.1 Momentum Update. As in [Hyde et al. 2020], the grid momentum update is derived from Equation (8):

$$
\begin{aligned}
m_{\mathbf{i}}^{n} \frac{\hat{\mathbf{v}}_{\mathbf{i}}^{n+1}-\mathbf{v}_{\mathbf{i}}^{n}}{\Delta t} & =\mathbf{f}_{\mathbf{i}}(\mathbf{x}+\Delta t \hat{\mathbf{q}})+m_{\mathbf{i}}^{n} \mathbf{g}, \\
\mathbf{f}_{\mathbf{i}}(\hat{\mathbf{x}}) & =-\frac{\partial e}{\partial \hat{\mathbf{x}}_{\mathbf{i}}}(\hat{\mathbf{x}})-\mu^{l} \sum_{p} \boldsymbol{\epsilon}^{v}\left(\hat{\mathbf{x}} ; \mathbf{x}_{p}^{n}\right)\left(\frac{\partial N_{\mathbf{i}}}{\partial \mathbf{x}}\left(\mathbf{x}_{p}^{n}\right)\right)^{T} V_{p}^{n},
\end{aligned}
$$

where $\mathbf{f}_{\mathbf{i}}$ is the force on grid node $\mathbf{i}$ from potential energy and viscosity, $\epsilon^{v}\left(\hat{\mathbf{x}} ; \mathbf{x}_{p}^{n}\right)=\frac{1}{2}\left(\sum_{\mathbf{j}} \hat{\mathbf{x}}_{\mathbf{j}} \frac{\partial N_{\mathbf{j}}}{\partial \mathbf{x}}\left(\mathbf{x}_{p}^{n}\right)+\left(\hat{\mathbf{x}}_{\mathbf{j}} \frac{\partial N_{\mathbf{j}}}{\partial \mathbf{x}}\left(\mathbf{x}_{p}^{n}\right)\right)^{T}\right)$ is the strain rate at $\mathrm{x}_{p}^{n}, \mathrm{~g}$ is gravity, and $\hat{\mathrm{q}}$ is either 0 (for explicit time integration) or $\hat{\mathbf{v}}^{n+1}$ (for backward Euler time integration). $\mathbf{x}$ represents the vector of all unmoved grid node positions $\mathbf{x}_{\mathbf{i}}$. We use $e(\mathbf{y})$ to denote the discrete potential energy $\Psi$ where MPM and surface particles are used as quadrature points:

$$
\begin{aligned}
e(\mathbf{y}) & =\sum_{p}\left(\psi^{h}\left(\mathbf{F}_{p}(\hat{\mathbf{y}})\right)+\frac{\lambda^{l}}{2}\left(J_{p}(\hat{\mathbf{y}})-1\right)^{2}\right) V_{p}^{0} \\
& +\sum_{r} k^{\sigma}\left(\mathbf{s}_{r}^{n}\right)\left|\hat{J}_{r}(\hat{\mathbf{y}}) \hat{\mathbf{F}}_{r}^{-T}(\hat{\mathbf{y}}) d \mathbf{A}_{r}^{n}\right|,
\end{aligned}
$$

where, as in [Stomakhin et al. 2013], $\mathbf{F}_{p}(\hat{\mathbf{y}})=\sum_{\mathbf{i}} \mathbf{y}_{\mathbf{i}} \frac{\partial N_{\mathrm{i}}}{\partial \mathbf{x}}\left(\mathbf{x}_{p}^{n}\right) \mathbf{F}_{p}^{n}$ and as in [Hyde et al. 2020], $J_{p}(\hat{\mathbf{y}})=\left(1-d+y_{\alpha} \frac{\partial N_{\mathrm{i}}}{\partial x_{\alpha}}\left(\mathbf{x}_{p}^{n}\right)\right) J_{p}^{n}$ and $\hat{\mathbf{F}}_{p}(\mathbf{y})=$ $\sum_{\mathrm{i}} \mathrm{y}_{\mathrm{i}} \frac{\partial N_{\mathrm{i}}}{\partial \mathrm{x}}\left(\mathrm{x}_{p}^{n}\right)$. With these conventions, the $\alpha$ component of the energy-based force on grid node $\mathbf{i}$ is of the form

$$
\begin{aligned}
-\frac{\partial e}{\partial x_{\mathrm{i} \alpha}}(\mathrm{y})= & -\sum_{p} \frac{\partial \psi^{h}}{\partial F_{\alpha \delta}}\left(\mathrm{F}_{p}(\hat{\mathbf{y}})\right) F_{p \gamma \delta}^{n} \frac{\partial N_{\mathrm{i}}}{\partial x_{\gamma}}\left(\mathbf{x}_{p}^{n}\right) V_{p}^{0} \\
& -\sum_{p} \lambda^{l}\left(J_{p}(\mathbf{y})-1\right) \frac{\partial N_{\mathrm{i}}}{\partial x_{\alpha}}\left(\mathbf{x}_{p}^{n}\right) J_{p}^{n} V_{p}^{0} \\
& -\sum_{r} k^{\sigma}\left(\mathbf{s}_{r}^{n}\right) \frac{\partial\left|\operatorname{det}\left(\hat{\mathbf{F}}_{r}\right) \hat{\mathbf{F}}_{r}^{-T} d \mathrm{~A}_{r}^{n}\right|}{\partial \hat{F}_{\alpha \delta}}\left(\hat{\mathbf{F}}_{r}(\hat{\mathbf{y}})\right) \frac{\partial N_{\mathrm{i}}}{\partial x_{\delta}}\left(\mathbf{x}_{p}^{n}\right) .
\end{aligned}
$$

We note that the viscous contribution to the force in Equation (15) is the same as in [Ram et al. 2015]. We would expect $V_{p}^{n+1}$ in this 
term when deriving from Equation (9), however we approximate it as $V_{p}^{n}$. This is advantageous since it makes the term linear; and since $\hat{J}_{p}(\hat{\mathbf{x}}) \approx 1$ from the liquid pressure and hyperelastic stress, it is not a poor approximation. Lastly, we note that the surface tension coefficient $k^{\sigma}\left(\mathbf{s}_{r}^{n}\right)$ will typically get its spatial dependence from composition with a function of temperature $k^{\sigma}\left(\mathbf{s}_{r}^{n}\right)=\tilde{k}^{\sigma}\left(\hat{T}\left(\mathbf{s}_{r}^{n}\right)\right)=$ $\tilde{k}^{\sigma}\left(\mathbf{T}_{r}^{s, n}\right)$.

In the case of implicit time stepping with backward Euler $(\hat{\mathrm{q}}=$ $\left.\hat{\mathbf{v}}^{n+1}\right)$, we use Newton's method to solve the nonlinear systems of equations. This requires linearization of the grid forces associated with potential energy in Equation (16). We refer the reader to [Stomakhin et al. 2013] and [Hyde et al. 2020] for the expressions for these terms, as well as the definiteness fix used for surface tension contributions.

4.3.2 Temperature Update. We discretize Equation (10) in a similar manner which results in the following equations for the grid temperatures $T_{\mathbf{i}}$ :

$$
\begin{aligned}
c_{p} m_{\mathbf{i}} \frac{\hat{T}_{\mathbf{i}}^{n+1}-T_{\mathbf{i}}^{n}}{\Delta t}= & -\sum_{p} K \frac{\partial N_{\mathbf{i}}}{\partial x_{\alpha}}\left(\mathbf{x}_{p}^{n}\right) \hat{T}_{\mathbf{j}}^{n+1} \frac{\partial N_{\mathbf{j}}}{\partial x_{\alpha}}\left(\mathbf{x}_{p}^{n}\right) V_{p}^{n} \\
& -\sum_{r} h N_{\mathbf{i}}\left(\mathbf{s}_{r}^{n}\right) \hat{T}_{\mathbf{j}}^{n+1} N_{\mathbf{j}}\left(\mathbf{s}_{r}^{n}\right)\left|d \mathbf{A}_{r}^{n}\right| \\
& +\sum_{r} N_{\mathbf{i}}\left(\mathbf{s}_{r}^{n}\right)\left[h \bar{T}\left(\mathbf{s}_{r}^{n}\right)+b\left(\mathbf{s}_{r}^{n}\right)\right]\left|d \mathbf{A}_{r}^{n}\right| \\
& +\sum_{p} h N_{\mathbf{i}}\left(\mathbf{x}_{p}^{n}\right) H\left(\mathbf{x}_{p}^{n}\right) V_{p}^{n} .
\end{aligned}
$$

Note that by using the surface particles $\mathbf{s}_{r}^{n}$ as quadrature points in the variational form, the Robin boundary condition can be discretized naturally with minimal modification to the Laplacian and time derivative terms. Also, note that the inclusion of this term modifies both the matrix and the right side in the linear system for $\hat{T}_{i}^{n+1}$. We found that performing constant extrapolation of interior particle temperatures to the surface particles provided better initial guesses for the linear solver.

\section{TRANSFER: G2P}

Once grid momentum and temperature have been updated, we transfer velocity and temperature back to the particles. For interior MPM particles with no associated surface or balance particles $\left(\Pi_{p}=\right.$ $\varnothing)$, we transfer velocity, affine velocity and temperature from the grid to particles in the standard APIC [Jiang et al. 2015] way:

$$
\mathbf{v}_{p}^{n+1}=\sum_{\mathbf{i}} N_{\mathbf{i}}\left(\mathbf{x}_{p}^{n}\right) \hat{\mathbf{v}}_{\mathbf{i}}^{n+1}, \quad \mathbf{A}_{p}^{n+1}=\frac{4}{\Delta x^{2}} \sum_{\mathbf{i}} N_{\mathbf{i}}\left(\mathbf{x}_{p}^{n}\right) \hat{\mathbf{v}}_{\mathbf{i}}^{n+1}\left(\mathbf{x}_{\mathbf{i}}-\mathbf{x}_{p}^{n}\right)^{T} .
$$

For interior MPM particles that were split with a collection of surface and balance particles $\left(\Pi_{p} \neq \varnothing\right)$, more care must be taken since surface and balance particles will be deleted at the end of the time step. First, the particle is reassigned its initial mass $m_{p}$. Then we compute the portion of the grid momentum associated with each surface and balance particle associated with $p$ as

$$
\mathbf{p}_{\mathbf{i} r}^{s}=\tilde{m}_{p} N_{\mathbf{i}}\left(\mathbf{s}_{r}^{n}\right) \hat{\mathbf{v}}_{\mathbf{i}}^{n+1}, \mathbf{p}_{\mathbf{i} r}^{b}=\tilde{m}_{p} N_{\mathbf{i}}\left(\mathbf{b}_{r}^{n}\right) \hat{\mathbf{v}}_{\mathbf{i}}^{n+1}, r \in \Pi_{p} .
$$

We then sum this with the split particle's share of the grid momentum to define the merged particle's share of the grid momentum

$$
\mathrm{p}_{\mathbf{i} p}=\tilde{m}_{p} N_{\mathbf{i}}\left(\mathbf{x}_{p}^{n}\right) \hat{\mathbf{v}}_{\mathbf{i}}^{n+1}+\sum_{r \in \Pi_{p}} \mathbf{p}_{\mathbf{i} r}^{s}+\mathbf{p}_{\mathbf{i} r}^{b} .
$$

Note that the $\mathbf{p}_{\mathbf{i} p}$ may be nonzero for more grid nodes than the particle would normally splat to (see Figure 3). We define the particle velocity from the total momentum by dividing by the mass $\mathbf{v}_{p}^{n+1}=\frac{1}{m_{p}} \sum_{\mathbf{i}} \mathbf{p}_{\mathbf{i} p}$. To define the affine particle velocity, we use a generalization of [Fu et al. 2017] and first compute the generalized affine moments $t_{p \beta \gamma}=\sum_{\mathbf{i}} Q_{\mathbf{i} \alpha \beta \gamma} p_{\mathbf{i} p \alpha}$ of the momentum distribution $p_{\mathrm{i} p \alpha}$ where $Q_{\mathrm{i} \alpha \beta \gamma}=r_{\mathrm{i} p \gamma} \delta_{\alpha \beta}$ is the $\alpha$ component of the $\beta \gamma$ linear mode at grid node $\mathbf{i}$. Here $\mathbf{r}_{\mathbf{i} p}=\mathbf{x}_{\mathbf{i}}-\mathbf{x}_{p}^{n}$ is the displacement from the center of mass of the distribution to the grid node $\mathbf{x}_{\mathbf{i}}$. We note that these moments are the generalizations of angular momentum to affine motion, as was observed in [Jiang et al. 2015], however in our case we compute the moments from a potentially wider distribution of momenta $p_{\mathrm{i} p \alpha}$. Lastly, to conserve angular momenta (see [Chen et al. 2021] for details), we define the affine velocity by inverting the generalized affine inertia tensor $\sum_{\mathbf{i}} Q_{\mathbf{i} \alpha \gamma \delta} m_{p} N_{\mathbf{i}}\left(\mathbf{x}_{p}^{n}\right) Q_{\mathbf{i} \alpha \epsilon \tau}$ of the point $\mathbf{x}_{p}^{n}$ using its merged mass distribution $m_{p} N_{\mathbf{i}}\left(\mathbf{x}_{p}^{n}\right)$. However, as noted in [Jiang et al. 2015], the generalized inertia tensor $\frac{m_{p} \Delta x^{2}}{4} \mathbf{I}$ is constant diagonal when using quadratic B-splines for $N_{\mathbf{i}}\left(\mathbf{x}_{p}^{n}\right)$ and therefore the final affine velocity is $\mathrm{A}_{p}^{n+1}=\frac{4}{m_{p} \Delta x^{2}} \mathbf{t}_{p}$.

Temperature and temperature gradients are transferred in the same way whether or not a MPM particle was split or not:

$$
T_{p}^{n+1}=\sum_{\mathbf{i}} \hat{T}_{\mathbf{i}}^{n+1} N\left(\mathbf{x}_{\mathbf{i}}\right), \nabla T_{p}^{n+1}=\sum_{\mathbf{i}} \hat{T}_{\mathbf{i}}^{n+1} \nabla N\left(\mathbf{x}_{\mathbf{i}}\right) .
$$

\section{EXAMPLES}

\subsection{Conservation}

To demonstrate our method's ability to fully conserve momentum and center of mass, we simulate a two-dimensional ellipse that oscillates under zero gravity due to surface tension forces in a $1 \mathrm{~m} \times$ $1 \mathrm{~m}$ domain. The ellipse has semiaxes of $0.3 \mathrm{~m}$ and $0.1 \mathrm{~m}$. We compare these results to those obtained using the method of Hyde et al. [2020].

The total linear momentum $\sum_{\mathbf{i}} m_{\mathbf{i}} v_{\mathbf{i}}$ and the total angular momentum about the origin $\sum_{\mathbf{i}} x_{\mathbf{i}} \times m_{\mathbf{i}} v_{\mathbf{i}}$ are calculated on the grid. We also compute the center of mass velocity error $\frac{\sum_{\mathrm{i}} m_{\mathrm{i}} v_{\mathrm{i}}}{\sum_{p} m_{p}}$ and the center of mass drift relative to the domain size. As shown in Figure 7 and 8 , the present technique perfectly conserves total linear momentum, total angular momentum, and the center of mass of the ellipse, unlike the approach of Hyde et al. [2020].

\subsection{Thermal Boundary Conditions}

The Robin boundary condition allows for the realistic convective heat transfer between the object and the environment. While such effects may be approximated by a simplified Dirichlet or Neumann boundary condition, the Robin boundary condition simplifies the process, and it is crucial for simulating temperature-dependent effects, such as the resolidification of the liquid wax in Section 6.9. 
Table 1. Summary of the simulation parameters. Example-specific variable $k_{L G}^{\sigma}$ can be found in the corresponding section. The unit for the thermal conductivity $K$ is W/ $(\mathrm{m} \cdot \mathrm{K})$; the convective heat transfer coefficient $h$ has a unit of $\mathrm{W} /\left(\mathrm{m}^{2} \cdot \mathrm{K}\right)$; the unit of the boundary heating rate is W/ $\mathrm{m}^{2}$. The number of particles $/$ cells used in each example is listed in Table 2.

\begin{tabular}{|c|c|c|c|c|c|c|c|c|}
\hline Example & $\Delta t[\mathrm{~s}]$ & CFL & $\Delta x[\mathrm{~m}]$ & Bulk Modulus [Pa] & Density $\left[\mathrm{kg} / \mathrm{m}^{3}\right]$ & $k^{\sigma}[\mathrm{N} / \mathrm{m}]$ & $k^{\sigma}$ Variation & Heat Transfer Coefficients \\
\hline Conservation (2D, Explicit) & $1 \times 10^{-5}$ & 0.6 & $1 / 63$ & 4166.67 & 10 & 0.1 & constant & N/A \\
\hline Two Spheres (2D) & $1 \times 10^{-2}$ to $5 \times 10^{-5}$ & 0.6 & $1 / 127$ & 833333.33 & 1 & 0.1 & constant & $K=0.0025 / h=10,0 / b=0$ \\
\hline Rotating Heat Flux (2D) & $1 \times 10^{-2}$ to $5 \times 10^{-5}$ & 0.6 & $1 / 127$ & 833333.33 & 1 & 0.1 & constant & $K=0.01 / h=0.1,0 / h=5$ \\
\hline Droplet Impact & $1 \times 10^{-2}$ to $5 \times 10^{-5}$ & $0.3,0.6$ & $1 / 127$ & 83333.33 & 10 & $20,15,5,1,0.1,0.05$ & constant & N/A \\
\hline Droplets on Ramps & $1 \times 10^{-2}$ to $1 \times 10^{-4}$ & 0.6 & $1 / 63$ & 16666.67 & 10 & $1.0\left(k_{S L}^{\sigma}\right), k_{L G}^{\sigma}$ varies & piecewise-constant & $\mathrm{N} / \mathrm{A}$ \\
\hline Contact Angles & 0.0333 to $1 \times 10^{-4}$ & 0.6 & $1 / 127$ & 83333.33 & 1 & $2.0\left(k_{S L}^{\sigma}\right), k_{L G}^{\sigma \sigma}$ varies & piecewise-constant & $\mathrm{N} / \mathrm{A}$ \\
\hline Soap Droplet in Water & $1 \times 10^{-2}$ to $5 \times 10^{-5}$ & 0.6 & $1 / 127$ & 16666.67 & 1 & 0.5 (water), 0.01 (soap) & piecewise-constant & $\mathrm{N} / \mathrm{A}$ \\
\hline Wine Glass & $1 \times 10^{-2}$ to $1 \times 10^{-5}$ & 0.6 & $1 / 127$ & 16666.67 & 1 & $0.05\left(k_{S L}^{\sigma}\right), 0.015\left(k_{L G}^{\sigma}\right)$ & piecewise-constant & $\mathrm{N} / \mathrm{A}$ \\
\hline Candles & $1 \times 10^{-2}$ to $1 \times 10^{-4}$ & 0.6 & $1 / 127$ & 833333.33 & 10 & $0.05,0.1,0.2,0.4$ & constant & $K=0.1 / h=0.5 / b=50$ \\
\hline Candles (Letters) & $1 \times 10^{-2}$ to $1 \times 10^{-4}$ & 0.6 & $1 / 127$ & 83333.33 & 10 & 0.05 & constant & $K=0.1 / h=2.5 / b=100$ \\
\hline Lid-Driven Cavity (2D) & $1 \times 10^{-3}$ & 0.6 & $1 / 63$ & 416.67 & 10 & 1.0 & temperature-dependent & $K=0.1 / h=0 / b=0$ \\
\hline Droplet with Marangoni Effect & $1 \times 10^{-2}$ to $5 \times 10^{-5}$ & 0.6 & $1 / 127$ & 83333.33 & 1 & $0.5 \sim 2.0$ & temperature-dependent & $K=0.1 / h=0.1 / b=50$ \\
\hline
\end{tabular}
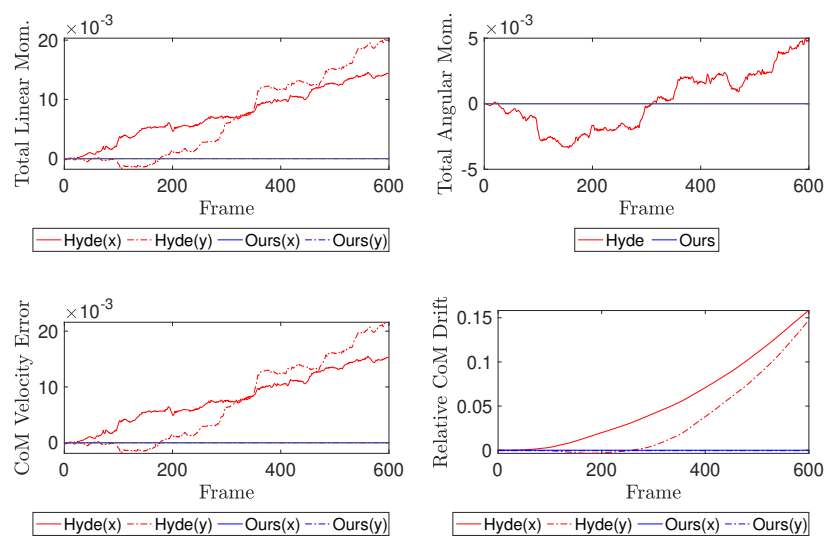

Fig. 7. The present method (blue) conserves total mass, total linear and angular momentum, and center of mass, unlike the method of Hyde et al. [2020] (red).
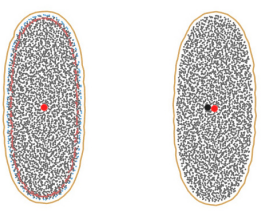

Ours

\begin{abstract}
Hyde
\end{abstract}

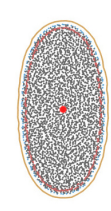

Ours

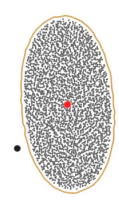

Hyde (a) $t=6.53 \mathrm{~s}$

(b) $t=18.9 \mathrm{~s}$

Fig. 8. An elliptical droplet oscillates under surface tension. The black dot indicates the initial location of the particles' center of mass, while the red dot is the position of the current center of mass. The drops in (a) are after 6 oscillation cycles, and the drops in (b) are after 18 cycles. The method of Hyde et al. [2020] does not conserve the momentum, so the center of mass drifts. Our method is conservative and preserves the center of mass even over a long period of time.

To demonstrate this effect, we initialize two discs side-by-side with radii of $0.15 \mathrm{~m}$. The domain size is $1 \mathrm{~m} \times 1 \mathrm{~m}$, and the ambient

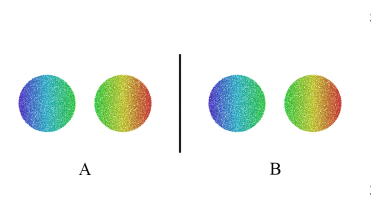

(a) $t=0 \mathrm{~s}$

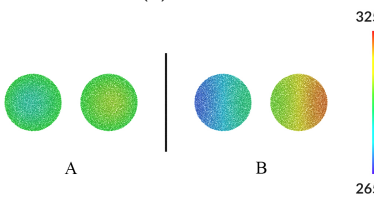

(c) $t=1 \mathrm{~s}$

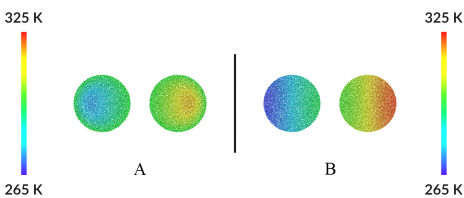

(b) $t=0.333 \mathrm{~s}$

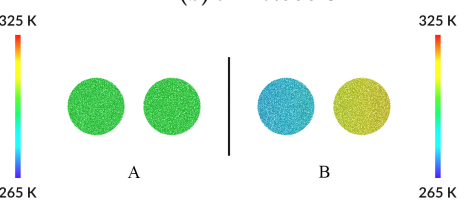

(d) $t=8 \mathrm{~s}$
Fig. 9. Heat transfer in two discs. The discs initially have linear temperature distribution. Simulation A has the Robin boundary condition applied, while simulation $B$ has only internal thermal conduction. The temperature in each disc reaches equilibrium over time. With the Robin boundary condition, the temperature of each disc approaches the ambient temperature.

temperature is $295 \mathrm{~K}$. The center of the left disc is at $(0.3,0.5)$, and the temperature increases linearly from $265 \mathrm{~K}$ at $x=0.15$ to $295 \mathrm{~K}$ at $x=0.45$. The center of the right one is at $(0.7,0.5)$, and the temperature increases linearly from $295 \mathrm{~K}$ at $x=0.55$ to $325 \mathrm{~K}$ at $x=0.85$. The thermal conductivity for both simulation $\mathrm{A}$ and $\mathrm{B}$ is $0.0025 \mathrm{~W} /(\mathrm{m} \cdot \mathrm{K})$. Simulation A has $h=10 \mathrm{~W} /\left(\mathrm{m}^{2} \cdot \mathrm{K}\right)$, while $h=0$ for simulation B. As shown in Figure 9, the Robin boundary condition equilibrates the temperature of the discs in simulation $\mathrm{A}$ to the ambient temperature, while in simulation $\mathrm{B}$, only the temperature in each disc reaches equilibrium.

Our method allows complex time-dependent boundary conditions to be applied. We simulate in Figure 10 a solid disc with a radius of $0.15 \mathrm{~m}$ with a heat flux of $b=5 \mathrm{~W} / \mathrm{m}^{2}$ applied on a section of its boundary. The disc is in a $1 \mathrm{~m} \times 1 \mathrm{~m}$ domain with the ambient temperature of $295 \mathrm{~K}$. The location heat flux rotates about the center of the disc at $2 \pi \mathrm{rad} / \mathrm{s}$. Simulation A has the Robin boundary condition $h=0.1 \mathrm{~W} /(\mathrm{m} \cdot \mathrm{K})$ applied, so the region without heat flux applied cools the disc to the ambient temperature. Simulation B does not have the Robin boundary condition, and the heat accumulates inside the disc. 


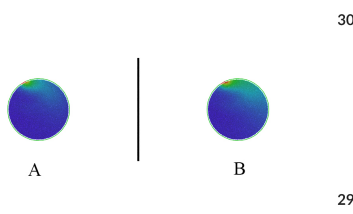

(a) $t=0.667 \mathrm{~s}$

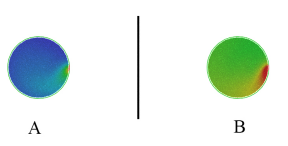

(c) $t=4 \mathrm{~s}$
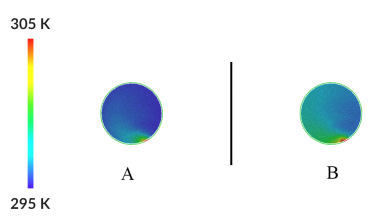

(b) $t=1.667 \mathrm{~s}$
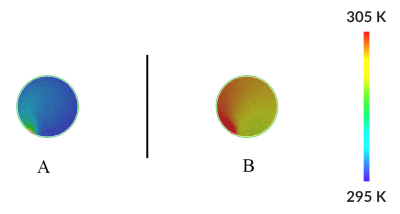

(d) $t=5.333 \mathrm{~s}$

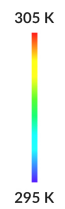

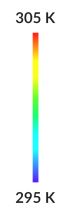

Fig. 10. Constant heat flux is applied to a small section of the disc boundary. The location of the heat flux rotates about the center of the disc at a constant speed. Robin boundary condition is enabled in simulation A and disabled in simulation B.

\subsection{Droplet Impact on Dry Surface}

Our method is able to handle highly dynamic simulations with a wide range of surface tension strengths. We simulate several inviscid spherical droplets with radii of $0.15 \mathrm{~m}$ free fall and impact a dry, frictionless, hydrophobic surface. In the top comparison of Figure 11 , droplets with different surface tension coefficients drop from a height of $2.5 \mathrm{~m}$. The size of the simulation domain is $1 \mathrm{~m} \times 3 \mathrm{~m} \times 1 \mathrm{~m}$. With different surface tension coefficients $k^{\sigma}$, the droplets display distinct behaviors upon impact, as shown in Figure 11.

We also capture the partial rebound and the full rebound behaviors of the droplet after the impact. The middle and bottom rows of Figure 11 show the footage of a droplet with $k^{\sigma}=15 \mathrm{~N} / \mathrm{m}$ dropped from a height of $3.5 \mathrm{~m}$ (in $1 \mathrm{~m} \times 4 \mathrm{~m} \times 1 \mathrm{~m}$ domain) and a droplet with $k^{\sigma}=5 \mathrm{~N} / \mathrm{m}$ dropped from a height of $2.5 \mathrm{~m}$ (in $1 \mathrm{~m} \times 3 \mathrm{~m} \times 1 \mathrm{~m}$ domain), respectively. With a higher surface tension coefficient and a higher impact speed, the droplet is able to completely leave the surface after the impact. Our results qualitatively match the experiment outcomes from [Rioboo et al. 2001].

\subsection{Droplets on Ramps}

As discussed in Section 3.6, our method allows for distinct $k^{\sigma}$ values at solid-liquid and liquid-air interfaces. Tuning the ratio between $k^{\sigma}$ at these interfaces allows simulating different levels of hydrophilicity/hydrophobicity. Figure 12 shows an example of several liquid drops with different $k^{\sigma}$ ratios falling on ramps of $5.5^{\circ}$ angle. The length of the ramp is $3 \mathrm{~m}$, and the domain size is $3 \mathrm{~m} \times 0.5 \mathrm{~m} \times 1 \mathrm{~m}$ Coulomb friction with a friction coefficient of 0.2 was used for the ramp surface, and the drop has no viscosity. When there is a larger difference between solid-liquid and liquid-air surface tension coefficients (i.e., a smaller $k^{\sigma}$ ratio), the liquid tends to drag more on the surface and undergo more separation and sticking. The leftmost example, with a $k^{\sigma}$ ratio of 1.0 , exhibits hydrophobic behavior.

\subsection{Lid-Driven Cavity}

The two-dimensional lid-driven cavity is a classic example in the engineering literature of the Marangoni effect [Francois et al. 2006;

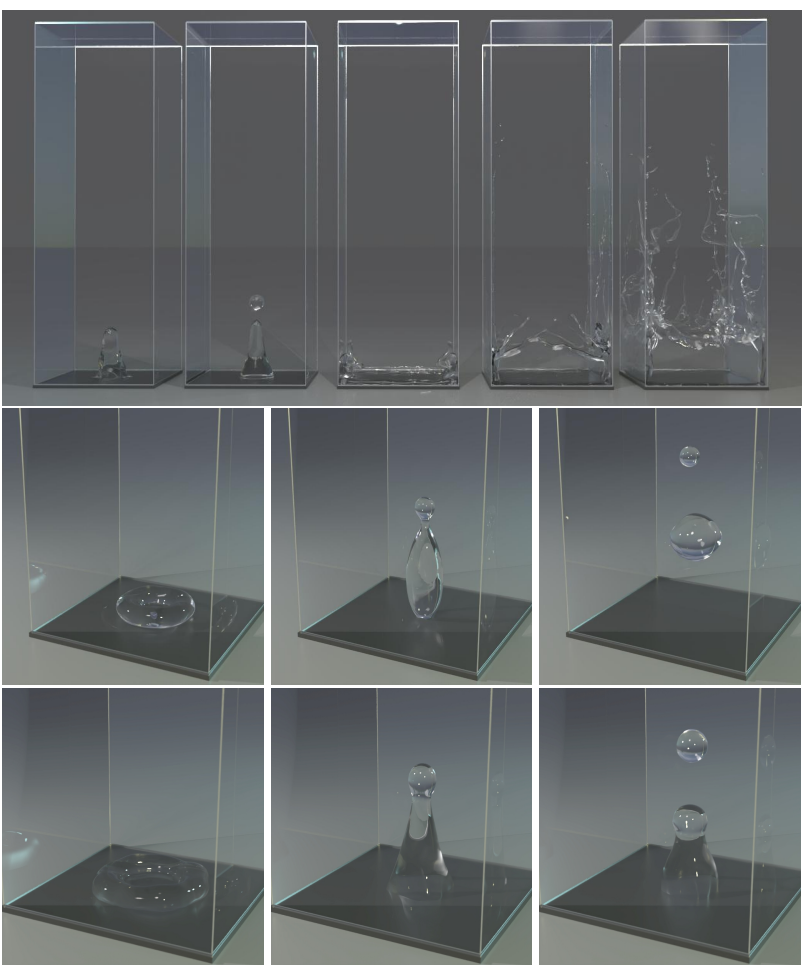

Fig. 11. (Top) Spherical droplets with different surface tension coefficients free fall from the same height. In the top figure, from left to right, the surface tension coefficients are $k^{\sigma}=20,5,1,0.1,0.05 \mathrm{~N} / \mathrm{m}$. (Middle Row) full rebound of the droplet (initial height: $3.5 \mathrm{~m}$ and $k^{\sigma}=15 \mathrm{~N} / \mathrm{m}$ ). (Bottom Row) partial rebound of the droplet (initial height: $2.5 \mathrm{~m}$ and $k^{\sigma}=5 \mathrm{~N} / \mathrm{m}$ ).

Hopp-Hirschler et al. 2018]. Inspired by works like these, we simulate a square unit domain and fill the domain with particles up to height $1-4 \Delta x(\Delta x=1 / 63)$, which results in a free surface near the top of the domain. A linear temperature gradient from 1 on the left to 0 on the right is initialized on the particles. To achieve the Marangoni effect, the surface tension coefficient $k^{\sigma}$ is set to depend linearly on temperature: $k^{\sigma}=1-T_{p} . k^{\sigma}$ is clamped to be in $[0,1]$ to avoid artifacts due to numerical precision. Gravity is set to zero, dynamic viscosity is set to $1 \times 10^{-6} \mathrm{~Pa} \cdot \mathrm{m}$ and implicit MPM is used with a maximum $\Delta t$ of 0.001 . Results are shown in Figure 13 . We note that the center of the circulation drifts to the right over the course of the simulation due to uneven particle distribution resulting from the circulation of the particles; this drifting behavior is not observed in works like [Francois et al. 2006] or [Hopp-Hirschler et al. 2018]. Investigating particle reseeding strategies to stabilize the flow is interesting future work.

\subsection{Contact Angles}

Figure 14 shows that our method enables simulation of various contact angles, emulating various degrees of hydrophobic or hydrophilic behavior as a droplet settles on a surface. We adjust the contact angles by assigning one surface tension coefficient, $k_{L G}^{\sigma}$, to the surface particles on the liquid-gas interface, and another one, 
Table 2. Performance measurements for one time step of several of our 3D examples, broken down by (1) sampling: generating surface and balance particles and conservative momentum splitting, (2) conservative momentum merging, (3) single particle-to-grid transfer, (4) single grid-to-particle transfer, (5) total time of the linear solve, (6) total time of one time step. Note that each linear solve involves several particle-to-grid and grid-to-particle transfers, and each time step requires several linear solves. All times are in milliseconds.

\begin{tabular}{|c|c|c|c|c|c|c|c|c|c|}
\hline Example & \# Cells & \# Int. Part. & \# Surf. Part. & Sampling & Merging & Part. $\rightarrow$ Grid & Grid $\rightarrow$ Part. & Linear Solve & Time Step \\
\hline Droplet Impact $\left(k^{\sigma}=5\right)$ & $2 \mathrm{M}$ & $794 \mathrm{~K}$ & $100 \mathrm{~K}$ & 2224 & 20 & 95 & 39 & 1422 & 10065 \\
\hline Droplets on Ramps $\left(k_{S L}^{\sigma} / k_{L G}^{\sigma}=0.05\right)$ & $1.5 \mathrm{M}$ & $70 \mathrm{~K}$ & $100 \mathrm{~K}$ & 258 & 6 & 28 & 9 & 199 & 1434 \\
\hline Contact Angles $\left(k_{S L}^{\sigma} / k_{L G}^{\sigma}=0\right)$ & $256 \mathrm{~K}$ & $230 \mathrm{~K}$ & $250 \mathrm{~K}$ & 492 & 17 & 73 & 38 & 647 & 4286 \\
\hline Soap Droplet in Water & $1 \mathrm{M}$ & $4 \mathrm{M}$ & $200 \mathrm{~K}$ & 2166 & 33 & 575 & 257 & 5599 & 35304 \\
\hline Wine Glass & $2 \mathrm{M}$ & $1.6 \mathrm{M}$ & $500 \mathrm{~K}$ & 1549 & 52 & 163 & 91 & 2030 & 12440 \\
\hline Candle $\left(k^{\sigma}=0.1\right)$ & $2 \mathrm{M}$ & $618 \mathrm{~K}$ & $50 \mathrm{~K}$ & 1420 & 7 & 122 & 44 & 2646 & 29162 \\
\hline Candle Letters & $256 \mathrm{~K}$ & $3.1 \mathrm{M}$ & $100 \mathrm{~K}$ & 4601 & 15 & 574 & 187 & 8445 & 172787 \\
\hline Droplet with Marangoni Effect & $4.1 \mathrm{M}$ & $235 \mathrm{~K}$ & $200 \mathrm{~K}$ & 2991 & 18 & 82 & 51 & 1636 & 12056 \\
\hline
\end{tabular}

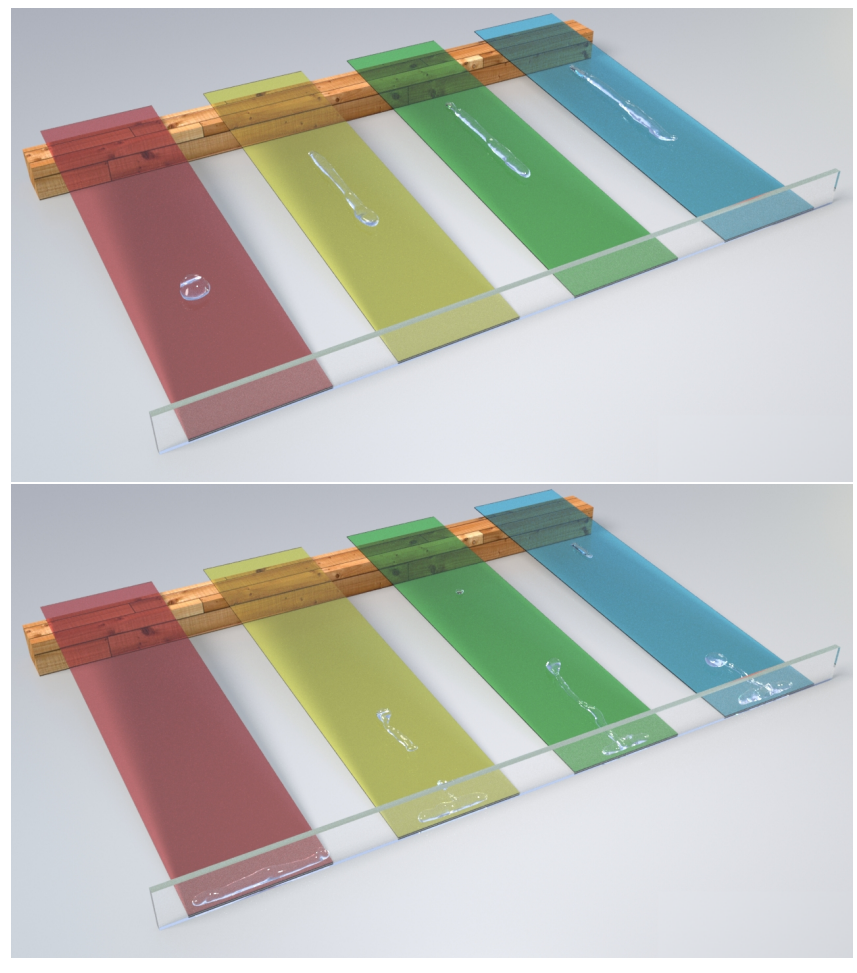

Fig. 12. Liquid drops fall on a ramp with varying ratios between the solidliquid and liquid-air surface tension coefficients. From left to right: ratios of 1.0, 0.6, 0.3, 0.05. (Top) Frame 60. (Bottom) Frame 100.

$k_{S L}^{\sigma}$, to those on the solid-liquid interface. Following the Young equation (Equation (11)) and our assumption that $k_{S G}^{\sigma}$ is negligible, the contact angle is given by $\theta=\arccos \left(-k_{S L}^{\sigma} / k_{L G}^{\sigma}\right)$. Note that the effect of gravity will result in contact angles slightly smaller than targeted.

A droplet of radius $0.1 \mathrm{~m}$ is placed right above the ground in a $0.5 \mathrm{~m} \times 0.5 \mathrm{~m} \times 0.5 \mathrm{~m}$ domain. Each droplet is discretized using $230 \mathrm{k}$ interior particles and 250k surface particles. The surface tension $k_{L G}^{\sigma}$ is set to $2 \mathrm{~N} / \mathrm{m}$, and we approximate $k_{S L}^{\sigma}$ based on the desired

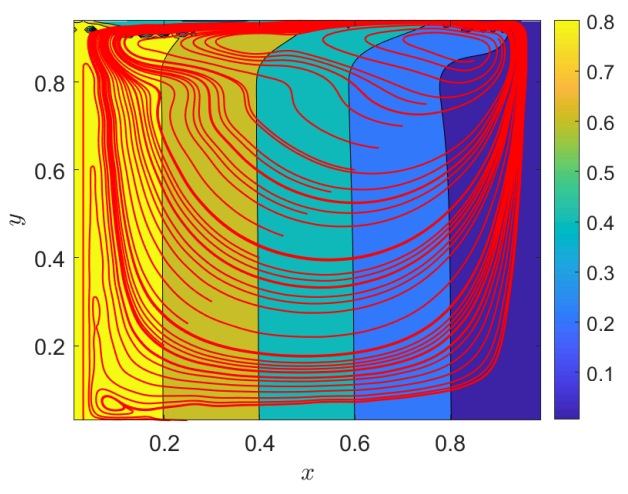

Fig. 13. Frame 500 of a two-dimensional lid-driven cavity simulation. The simulation is initially stationary, but velocity streamlines (red) show the flow pattern characteristic of Marangoni convection that develops due to a temperature-dependent surface tension coefficient. The contour plot shows the evolving temperature field (initially a linear horizontal distribution).

contact angle $\theta$. A dynamic viscosity of $0.075 \mathrm{~Pa} \cdot \mathrm{s}$ is used to stabilize the simulations.
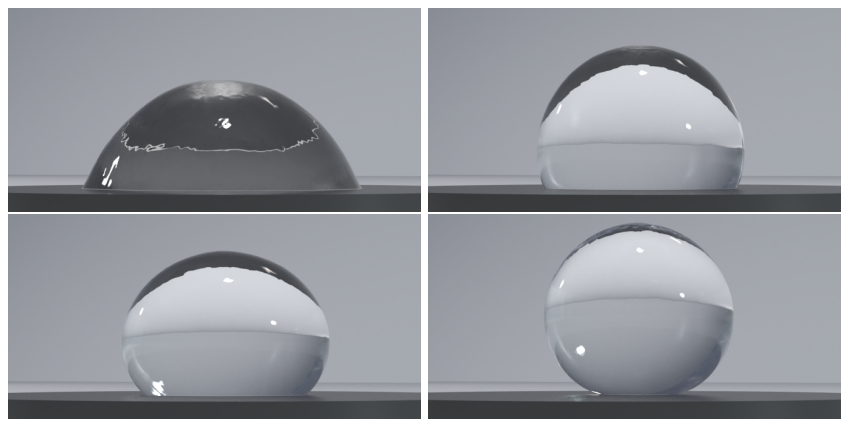

Fig. 14. As our droplets settle, we are able to obtain contact angles of approximately $45,90,135$ and 180 degrees, using a $k_{S L}^{\sigma} / k_{L G}^{\sigma}$ ratio of $-\sqrt{2} / 2,0$, $\sqrt{2} / 2$ and 1 , respectively. 


\subsection{Soap Droplet in Water}

We demonstrate a surface tension driven flow by simulating soap reducing the surface tension of the water. We initialize a $1 \mathrm{~m} \times$ $0.05 \mathrm{~m} \times 1 \mathrm{~m}$ rectangular water pool, set a $0.075 \mathrm{~m}$-radius and $0.025 \mathrm{~m}$ height cylindrical region at the center of the pool to be liquid soap and identify particles in this region to be soap particles. We set the surface tension coefficients for the surface particles based on the type of its closest MPM particles. Surface particles associated with the water particles have higher surface tension than those associated with the soap particles. The viscosity is set to 0 in this example.

In order to visualize the effect of the surface tension driven flow, we randomly selected marker particles on the top surface of the pool. Due to the presence of the soap, the center of the pool has lower surface tension than the area near the edge of the container. The surface tension gradient drives the particles to flow from the center to the edge. Figure 15 shows footage of this process.
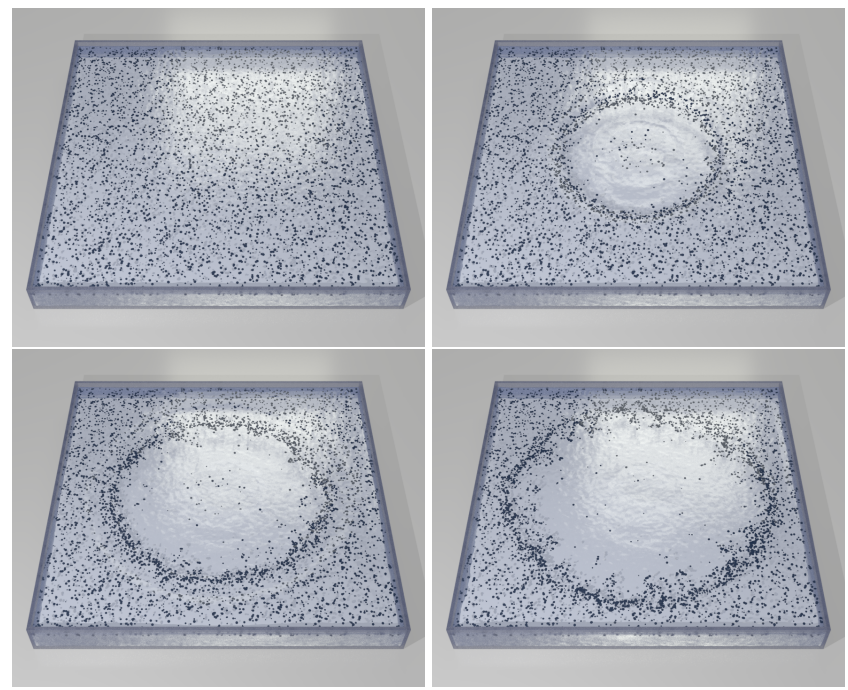

Fig. 15. The soap in the center of the pool surface reduces the surface tension. The surface tension gradient drives the markers towards the walls of the container. Frames 0, 10, 20, 40 are shown in this footage.

\subsection{Wine Glass}

We consider an example of wine flowing on the surface of a prewetted glass. The glass is an ellipsoid centered at $(0.5 \mathrm{~m}, 0.7 \mathrm{~m}, 0.5 \mathrm{~m})$ with characteristic dimensions $a=0.4 \mathrm{~m}, b=0.6 \mathrm{~m}$, and $c=0.4 \mathrm{~m}$. We initialize a thin band of particles with the thickness of $2 \Delta x \mathrm{~m}$ on the surface of the wine glass and observe the formation of ridges and fingers as the particles settle toward the bulk fluid in the glass. We set the surface tension coefficient on the liquid-gas interface to be $k_{L G}^{\sigma}=0.05 \mathrm{~N} / \mathrm{m}$ and the one on the solid-liquid interface to be $k_{S L}^{\sigma}=0.015 \mathrm{~N} / \mathrm{m}$. The piecewise constant surface tension leads to a more prominent streaking behavior of the liquid on the glass wall. The results are shown in Figure 16.
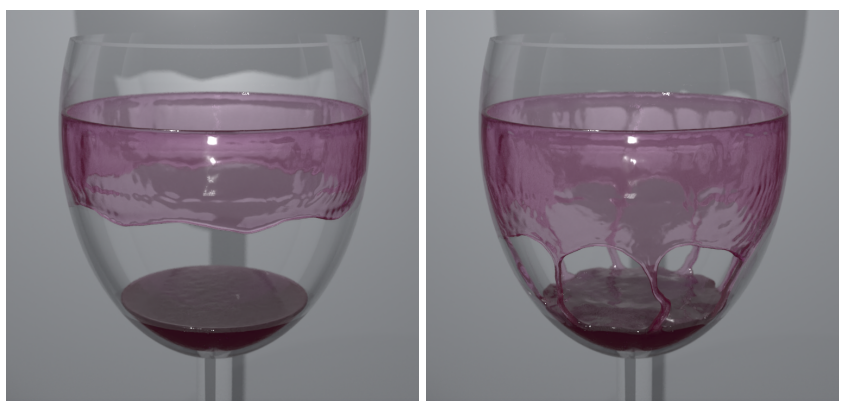

Fig. 16. Wine is initialized in a glass with part of the interior pre-wetted. The falling wine forms tears and ridges, and the tears eventually connect with the bulk fluid. Frames 30 and 90 are shown.

\subsection{Candles}

We simulate several scenarios with wax candles. The height of the candle is $0.6 \mathrm{~m}$ and the radius is $0.1 \mathrm{~m}$. The domain size is $1 \mathrm{~m} \times$ $1 \mathrm{~m} \times 1 \mathrm{~m}$. In these examples, wax melts due to a heat source (candle flame) and resolidifies when it flows away from the flame. Ambient temperature $\hat{T}$ is taken to be $298 \mathrm{~K}$, and the melting point is $303 \mathrm{~K}$. Thermal conductivity $K$ is taken to be $0.1 \mathrm{~W} / \mathrm{m} \cdot \mathrm{K}$, and specific heat capacity $c_{p}$ is set to $1 \mathrm{~J} / \mathrm{K}$. No internal heat source is used $(H=0)$; instead, heating and cooling are applied only via the boundary conditions.

To simulate the candle wicks, we manually construct and sample points on cubic splines. As the simulation progresses, we delete particles from the wick that are too far above the highest ( $y$-direction) liquid particle within a neighborhood of the wick. The flames are created by running a separate FLIP simulation as a postprocess and anchoring the result to the exposed portion of each wick. We rendered these scenes using Arnold [Georgiev et al. 2018] and postprocessed the renders using the NVIDIA OptiX denoiser (based on [Chaitanya et al. 2017]).

We consider the effect of varying $k^{\sigma}$ on the overall behavior of the flow. Figure 4 compares $k^{\sigma}$ values of $0.05 \mathrm{~N} / \mathrm{m}, 0.1 \mathrm{~N} / \mathrm{m}, 0.2 \mathrm{~N} / \mathrm{m}$, and $0.4 \mathrm{~N} / \mathrm{m}$. In these examples, a grid resolution of $\Delta x=1 / 127 \mathrm{~m}$ was used, along with boundary condition parameters $h=0.5 \mathrm{~W} / \mathrm{m}^{2} \cdot \mathrm{K}$ and $b=50 \mathrm{~W} / \mathrm{m}^{2}$. The figure demonstrates that as surface tension increases, the molten wax spreads significantly less. As the wax cools and resolidifies, visually interesting layering behavior is observed.

Figure 17 shows an example of several candle letters melting in a container. Wicks follow generally curved paths inside the letters. Melt pools from the different letters seamlessly interact. This simulation used a surface tension coefficient $k^{\sigma}=0.05 \mathrm{~N} / \mathrm{m}$, $h=2.5 \mathrm{~W} / \mathrm{m}^{2} \cdot \mathrm{K}, b=100 \mathrm{~W} / \mathrm{m}^{2}$, dynamic viscosity of $0.01 \mathrm{~Pa} \cdot \mathrm{s}$, $\Delta x=1 / 127 \mathrm{~m}$, and a bulk modulus of $83333.33 \mathrm{~Pa}$ for liquid and solid phases.

\subsection{Droplet with Marangoni Effect}

We simulate an inviscid liquid metal droplet that moves under the Marangoni effect, i.e., due to a temperature-induced surface tension gradient. A spherical drop with radius of $0.1 \mathrm{~m}$ is initialized at position $(0.5 \mathrm{~m},(0.1+3.5 \Delta x) \mathrm{m}, 0.5 \mathrm{~m})$ inside a $2 \mathrm{~m} \times 1 \mathrm{~m} \times 1 \mathrm{~m}$ domain. We then turn on the heating 1 second after the simulation starts (while 


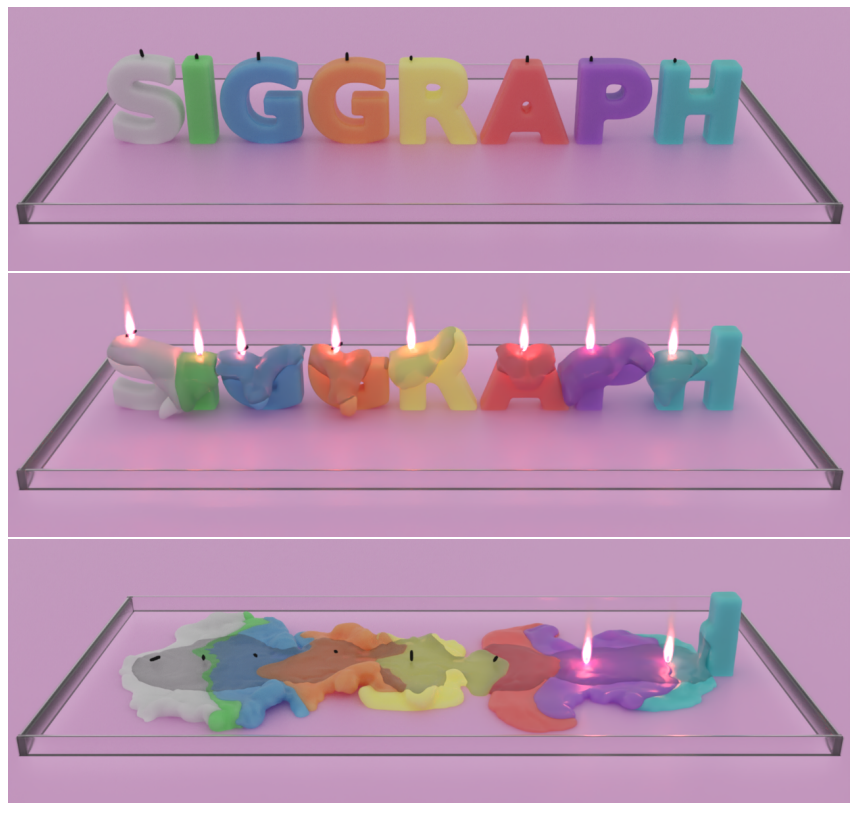

Fig. 17. Letter-shaped candles melt inside a container. (Top) Frame 1, before flames are lit. (Middle) Frame 60, in the middle of melting. (Bottom) Frame 200 , as flames are extinguished and wax pools resolidify.

the droplet is still spreading). The Neumann boundary condition is applied to heat the particles in the region with $x \leq 0.5 \mathrm{~m}$. The thermal conductivity $K$ is set to be $0.1 \mathrm{~W} /(\mathrm{m} \cdot \mathrm{K})$, and the convective heat transfer coefficient $h$ is $0.1 \mathrm{~W} /\left(\mathrm{m}^{2} \cdot \mathrm{K}\right)$. The boundary heating rate $b=50 \mathrm{~W} / \mathrm{m}^{2}$ is much higher than the conduction and convection, so the heat transfer inside the droplet and the heat exchange between the droplet and the environment are less prominent.

We define the surface tension coefficient as a function of temperature: $k^{\sigma}=\min (0.09(T-\bar{T})+0.5,2) \mathrm{N} / \mathrm{m}$, where $\bar{T}=50 \mathrm{~K}$ is the ambient temperature. At its original temperature, the surface tension coefficient $k^{\sigma}$ of the droplet is $0.5 \mathrm{~N} / \mathrm{m}$. As the temperature increases, $k^{\sigma}$ increases linearly with the temperature. The maximum allowable surface tension strength is $2 \mathrm{~N} / \mathrm{m}$. After the heating, surface particles on the hotter side of the droplet have higher surface tension. The stronger surface tension penalizes the area changes and drives the particles to flow to the colder side, as shown in Figure 18. This surface tension gradient results in an interesting self-propelled behavior of the liquid metal droplet.

\subsection{Performance}

Table 2 shows average per-timestep runtime details for several of our examples. For this table, all experiments were run on a workstation equipped with 128GB RAM and with dual Intel ${ }^{\circledR}$ Xeon $^{\circledR}$ E5-2687W v4 CPUs at $3.00 \mathrm{Ghz}$.

\section{DISCUSSION AND FUTURE WORK}

Our method allows for simulation of surface tension energies with spatial gradients, including those driven by variation in temperature. Our MPM approach to the problem resolves many interesting
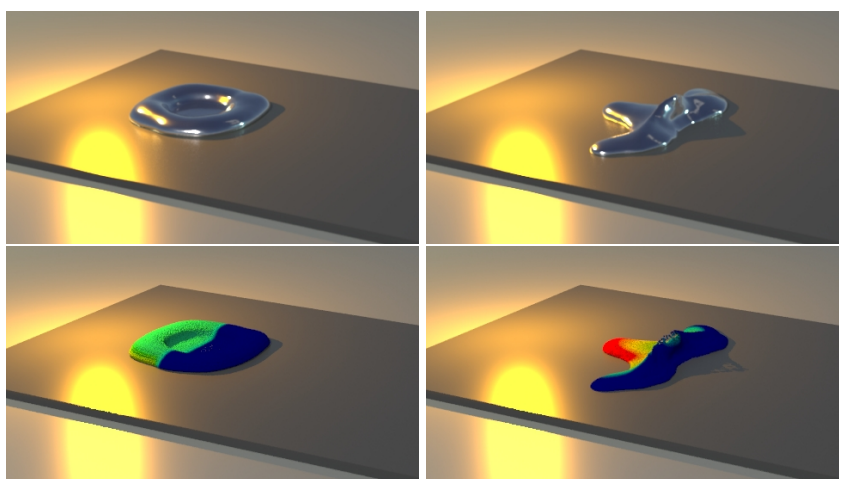

Fig. 18. A liquid metal droplet subjected to heating on one side. The surface tension coefficient increases as the temperature increases. (Top) the liquid metal at frame 45 and frame 130. (Bottom) the particle view of temperature distribution at frame 45 and frame 130. The red color indicates higher temperature.

characteristic phenomena associated with these variations. However, while we provide perfect conservation of linear and angular momentum, our approach to the thermal transfers is not perfectly conservative. Developing a thermally conservative transfer strategy is interesting future work. Also, although we simulate tears of wine on the walls of a glass, we did not simulate the effect of alcohol evaporation on the surface energy variation. Adding in a mixture model as in [Ding et al. 2019] would be interesting future work. Lastly, although our approach was designed for MPM, SPH is more commonly used for the simulation of liquids. However, SPH and MPM have many similarities, as recently shown by the work of Gissler et al. [2020b], and it would be interesting future work to generalize our approach to SPH.

\section{ACKNOWLEDGMENTS}

This research was supported by the Exascale Computing Project (17SC-20-SC), a collaborative effort of the U.S. Department of Energy Office of Science and the National Nuclear Security Administration. This research was also supported under DoE ORNL contract 4000171342. The authors thank Dr. Pirouz Kavehpour at UCLA for the valuable discussions and support.

\section{REFERENCES}

A. Adamson and A. Gast. 1967. Physical chemistry of surfaces. Vol. 150. Interscience Publishers New York.

R. Ando, N. Thurey, and R. Tsuruno. 2012. Preserving Fluid Sheets with Adaptively Sampled Anisotropic Particles. IEEE Trans Vis Comp Graph 18, 8 (Aug. 2012), 12021214.

O. Azencot, O. Vantzos, M. Wardetzky, M. Rumpf, and M. Ben-Chen. 2015. Functional thin films on surfaces. In Proc 14th ACM SIGGRAPH/Eurograph Symp Comp Anim. 137-146.

C. Batty, A. Uribe, B. Audoly, and E. Grinspun. 2012. Discrete viscous sheets. ACM Trans Graph (TOG) 31, 4 (2012), 1-7.

T. Belytschko, W. Liu, B. Moran, and K. Elkhodary. 2013. Nonlinear finite elements for continua and structures. John Wiley and sons.

J. Brackbill, D. Kothe, and C. Zemach. 1992. A continuum method for modeling surface tension. 7 Comp Phys 100, 2 (1992), 335-354.

G. Buscaglia and R. Ausas. 2011. Variational formulations for surface tension, capillarity and wetting. Comp Meth App Mech Eng 200, 45-46 (2011), 3011-3025.

A.B.D. Cassie and S. Baxter. 1944. Wettability of porous surfaces. Transactions of the Faraday society 40 (1944), 546-551. 
C. R. A. Chaitanya, A. S. Kaplanyan, C. Schied, M. Salvi, A. Lefohn, D. Nowrouzezahrai, and T. Aila. 2017. Interactive reconstruction of Monte Carlo image sequences using a recurrent denoising autoencoder. ACM Trans Graph 36, 4 (2017), 1-12.

J. Chen, V. Kala, A. Marquez-Razon, E. Gueidon, D. Hyde, and J. Teran. 2021. Supplementary Technical Document. Technical Report.

Y.-L. Chen, J. Meier, B. Solenthaler, and V.C. Azevedo. 2020. An Extended Cut-Cell Method for Sub-Grid Liquids Tracking with Surface Tension. ACM Trans Graph 39, 6, Article 169 (Nov. 2020), 13 pages. https://doi.org/10.1145/3414685.3417859

E. Chernyaev. 1995. Marching cubes 33: Construction of topologically correct isosurfaces Technical Report.

P. Clausen, M. Wicke, J. R. Shewchuk, and J. F. O'Brien. 2013. Simulating liquids and solid-liquid interactions with Lagrangian meshes. ACM Transactions on Graphics (TOG) 32, 2 (2013), 17.

M. Corsini, P. Cignoni, and R. Scopigno. 2012. Efficient and Flexible Sampling with Blue Noise Properties of Triangular Meshes. IEEE Trans Vis Comp Graph 18, 6 (2012), 914-924. https://doi.org/10.1109/TVCG.2012.34

F. Da, C. Batty, C. Wojtan, and E. Grinspun. 2015. Double bubbles sans toil and trouble: discrete circulation-preserving vortex sheets for soap films and foams. ACM Trans Graph (SIGGRAPH 2015) (2015).

F. Da, D. Hahn, C. Batty, C. Wojtan, and E. Grinspun. 2016. Surface-only liquids. ACM Trans Graph (TOG) 35, 4 (2016), 1-12.

G. Daviet and F. Bertails-Descoubes. 2016. A Semi-implicit Material Point Method for the Continuum Simulation of Granular Materials. ACM Trans Graph 35, 4 (2016) 102:1-102:13.

C. C. de Langavant, A. Guittet, M. Theillard, F. Temprano-Coleto, and F. Gibou. 2017 Level-set simulations of soluble surfactant driven flows. F Comp Phys 348 (2017), 271-297.

A. de Vaucorbeil, V. P. Nguyen, S. Sinaie, and J. Y. Wu. 2020. Chapter Two - Material point method after 25 years: Theory, implementation, and applications. Advances in Applied Mechanics, Vol. 53. Elsevier, 185 - 398. https://doi.org/10.1016/bs.aams. 2019.11.001

M. Ding, X. Han, S. Wang, T. Gast, and J. Teran. 2019. A thermomechanical material point method for baking and cooking. ACM Trans Graph 38, 6 (2019), 192.

Y. Dukler, H. Ji, C. Falcon, and A. L Bertozzi. 2020. Theory for undercompressive shocks in tears of wine. Phys Rev Fluids 5, 3 (2020), 034002

E. Edwards and R. Bridson. 2012. A high-order accurate Particle-In-Cell method. Int 7 Numer Meth Eng 90 (2012), 1073-1088.

Y. Fang, M. Li, Ming Gao, and Chenfanfu Jiang. 2019. Silly rubber: an implicit material point method for simulating non-equilibrated viscoelastic and elastoplastic solids. ACM Trans Graph 38, 4 (2019), 1-13.

R. Farahi, A. Passian, T. Ferrell, and T. Thundat. 2004. Microfluidic manipulation via Marangoni forces. Applied Phys Let 85, 18 (2004), 4237-4239.

Y. Fei, C. Batty, E. Grinspun, and C. Zheng. 2018. A multi-scale model for simulating liquid-fabric interactions. ACM Trans Graph 37, 4 (2018), 51:1-51:16. https://doi org/10.1145/3197517.3201392

Y. Fei, H. Maia, C. Batty, C. Zheng, and E. Grinspun. 2017. A multi-scale model for simulating liquid-hair interactions. ACM Trans. Graph. 36, 4 (2017), 56:1-56:17. https://doi.org/10.1145/3072959.3073630

M. M. Francois, J. M. Sicilian, and D. B. Kothe. 2006. Modeling of thermocapillary forces within a volume tracking algorithm. In Modeling of Casting, Welding and Advanced Solidification Processes-XI (Opio, France). 935-942.

C. Fu, Q. Guo, T. Gast, C. Jiang, and J. Teran. 2017. A Polynomial Particle-in-cell Method ACM Trans Graph 36, 6 (Nov. 2017), 222:1-222:12

M. Gao, A. Tampubolon, C. Jiang, and E. Sifakis. 2017b. An adaptive generalized interpolation material point method for simulating elastoplastic materials. $A C M$ Trans Graph 36, 6 (2017), 223:1-223:12. https://doi.org/10.1145/3130800.3130879

Y. Gao, S. Li, L. Yang, H. Qin, and A. Hao. 2017a. An efficient heat-based model for solid-liquid-gas phase transition and dynamic interaction. Graphical Models 94 (2017), 14 - 24. https://doi.org/10.1016/j.gmod.2017.09.001

I. Georgiev, T. Ize, M. Farnsworth, R. Montoya-Vozmediano, A. King, B. Van Lommel, A. Jimenez, O. Anson, S. Ogaki, E. Johnston, A. Herubel, D. Russell, F. Servant, and M. Fajardo. 2018. Arnold: A brute-force production path tracer. ACM Transactions on Graphics (TOG) 37, 3 (2018), 1-12.

C. Gissler, A. Henne, S. Band, A. Peer, and M. Teschner. 2020a. An Implicit Compressible SPH Solver for Snow Simulation. ACM Trans Graph 39, 4, Article 36 (July 2020), 16 pages. https://doi.org/10.1145/3386569.3392431

C. Gissler, A. Henne, S. Band, A. Peer, and M. Teschner. 2020b. An implicit compressible SPH solver for snow simulation. ACM Trans Graph (TOG) 39, 4 (2020), 36-1.

O. Gonzalez and A. Stuart. 2008. A first course in continuum mechanics. Cambridge University Press.

Y. Gu and Y.-H. Yang. 2016. Physics Based Boiling Bubble Simulation. In SIGGRAPH ASIA 2016 Technical Briefs (Macau) (SA '16). Association for Computing Machinery, New York, NY, USA, Article 5, 4 pages. https://doi.org/10.1145/3005358.3005385

Q. Guo, X. Han, C. Fu, T. Gast, R. Tamstorf, and J. Teran. 2018. A material point method for thin shells with frictional contact. ACM Trans Graph 37, 4 (2018), 147. https://doi.org/10.1145/3197517.3201346
F. Harlow and E. Welch. 1965. Numerical calculation of time-dependent viscous incompressible flow of fluid with free surface. Phys Fl 8, 12 (1965), 2182-2189.

H. Hochstetter and A. Kolb. 2017. Evaporation and Condensation of SPH-Based Fluids. In Proc ACM SIGGRAPH/Eurographics Symp Comp Anim (Los Angeles, California) (SCA '17). Association for Computing Machinery, New York, NY, USA, Article 3, 9 pages. https://doi.org/10.1145/3099564.3099580

M. Hopp-Hirschler, M. S. Shadloo, and U. Nieken. 2018. A Smoothed Particle Hydrodynamics approach for thermo-capillary flows. Comp Fluids 176 (2018), 1 - 19. https://doi.org/10.1016/j.compfluid.2018.09.010

H. Hu and P. Eberhard. 2017. Thermomechanically coupled conduction mode laser welding simulations using smoothed particle hydrodynamics. Comp Part Mech 4 (Oct 2017), 473-486. Issue 4. https://doi.org/10.1007/s40571-016-0140-5

W. Huang, J. Iseringhausen, T. Kneiphof, Z. Qu, C. Jiang, and M.B. Hullin. 2020. Chemomechanical Simulation of Soap Film Flow on Spherical Bubbles. ACM Trans Graph 39, 4, Article 41 (July 2020), 14 pages. https://doi.org/10.1145/3386569.3392094

D.A.B. Hyde, S.W. Gagniere, A. Marquez-Razon, and J. Teran. 2020. An Implicit Updated Lagrangian Formulation for Liquids with Large Surface Energy. ACM Trans Graph 39, 6, Article 183 (Nov. 2020), 13 pages. https://doi.org/10.1145/3414685.3417845

G. Irving, J. Teran, and R. Fedkiw. 2004. Invertible Finite Elements for Robust Simulation of Large Deformation. In Proc ACM SIGGRAPH/Eurograph Symp Comp Anim. 131140 .

S. Ishida, P. Synak, F. Narita, T. Hachisuka, and C. Wojtan. 2020. A Model for Soap Film Dynamics with Evolving Thickness. ACM Trans Graph 39, 4, Article 31 (July 2020), 11 pages. https://doi.org/10.1145/3386569.3392405

C. Jiang, T. Gast, and J. Teran. 2017. Anisotropic elastoplasticity for cloth, knit and hair frictional contact. ACM Trans Graph 36, 4 (2017), 152.

C. Jiang, C. Schroeder, A. Selle, J. Teran, and A. Stomakhin. 2015. The Affine ParticleIn-Cell Method. ACM Trans Graph 34, 4 (2015), 51:1-51:10.

C. Jiang, C. Schroeder, J. Teran, A. Stomakhin, and A. Selle. 2016. The Material Point Method for Simulating Continuum Materials. In ACM SIGGRAPH 2016 Course. 24:124:52.

R. E. Johnson Jr. and R. H. Dettre. 1964. Contact angle hysteresis. III. Study of an idealized heterogeneous surface. F Phys Chem 68, 7 (1964), 1744-1750.

G. Klár, T. Gast, A. Pradhana, C. Fu, C. Schroeder, C. Jiang, and J. Teran. 2016. Druckerprager Elastoplasticity for Sand Animation. ACM Trans Graph 35, 4 (2016), 103:1103:12.

D. Langbein. 2002. Capillary surfaces: shape - stability - dynamics, in particular under weightlessness. Vol. 178. Springer Science \& Business Media.

T. Lenaerts and P. Dutré. 2009. An architecture for unified SPH simulations. CW Reports (2009)

W. Li, D. Liu, M. Desbrun, J. Huang, and X. Liu. 2020. Kinetic-based Multiphase Flow Simulation. IEEE Trans Vis Comp Graph (2020).

F. Losasso, J. Talton, N. Kwatra, and R. Fedkiw. 2008. Two-Way Coupled SPH and Particle Level Set Fluid Simulation. IEEE Trans Visu Comp Graph 14, 4 (2008), 797-804.

T. Maeshima, Y. Kim, and T. I. Zohdi. 2020. Particle-scale numerical modeling of thermomechanical phenomena for additive manufacturing using the material point method. Computational Particle Mechanics (2020). https://doi.org/10.1007/s40571-020-00358-

J. Monaghan. 1992. Smoothed particle hydrodynamics. Ann Rev Astron Astroph 30, 1 (1992), 543-574.

R. Narain, A. Golas, and M. Lin. 2010. Free-flowing granular materials with two-way solid coupling. ACM Trans Graph 29, 6 (2010), 173:1-173:10.

S. Nas and G. Tryggvason. 2003. Thermocapillary interaction of two bubbles or drops. Int 7 Multiphase Flow 29, 7 (2003), 1117-1135. https://doi.org/10.1016/S0301-9322(03) 00084-3

R. Osada, T. Funkhouser, B. Chazelle, and D. Dobkin. 2002. Shape Distributions. ACM Trans. Graph. 21, 4 (Oct. 2002), 807-832. https://doi.org/10.1145/571647.571648

A. Paiva, F. Petronetto, T. Lewiner, and G. Tavares. 2009. Particle-based viscoplastic fluid/solid simulation. Computer-Aided Design 41, 4 (2009), 306-314.

M. Pauly, R. Keiser, B. Adams, P. Dutré, M. Gross, and L. Guibas. 2005. Meshless animation of fracturing solids. ACM Trans Graph 24, 3 (2005), 957-964. https: //doi.org/10.1145/1073204.1073296

S. Pirk, M. Jarząbek, T. Hädrich, D.L. Michels, and W. Palubicki. 2017. Interactive Wood Combustion for Botanical Tree Models. 36, 6, Article 197 (Nov. 2017), 12 pages. https://doi.org/10.1145/3130800.3130814

D. Ram, T. Gast, C. Jiang, C. Schroeder, A. Stomakhin, J. Teran, and P. Kavehpour. 2015. A material point method for viscoelastic fluids, foams and sponges. In Proc ACM SIGGRAPH/Eurograph Symp Comp Anim. 157-163.

R. Rioboo, C. Tropea, and M. Marengo. 2001. Outcomes from a Drop Impact on Solid Surfaces. Atomization and Sprays 11, 2 (2001).

M. A. Russell. 2018. A Smoothed Particle Hydrodynamics Model for the Simulation of Laser Fusion Additive Manufacturing Processes. Ph.D. Dissertation. UC Berkeley.

C. Schreck and C. Wojtan. 2020. A practical method for animating anisotropic elastoplastic materials. Computer Graphics Forum - Eurographics 2020 39, 2 (2020).

L. Scriven and C. Sternling. 1960. The marangoni effects. Nature 187, 4733 (1960), 186-188. 
A. Stomakhin, R. Howes, C. Schroeder, and J. Teran. 2012. Energetically consistent invertible elasticity. In Proc Symp Comp Anim. 25-32.

A. Stomakhin, C. Schroeder, L. Chai, J. Teran, and A. Selle. 2013. A Material Point Method for snow simulation. ACM Trans Graph 32, 4 (2013), 102:1-102:10.

A. Stomakhin, C. Schroeder, C. Jiang, L. Chai, J. Teran, and A. Selle. 2014. Augmented MPM for phase-change and varied materials. ACM Trans Graph 33, 4 (2014), 138:1138:11.

D. Sulsky, Z. Chen, and H. Schreyer. 1994. A particle method for history-dependent materials. Comp Meth App Mech Eng 118, 1 (1994), 179-196.

M. Sussman and M. Ohta. 2009. A stable and efficient method for treating surface tension in incompressible two-phase flow. SIAM 7 Sci Comp 31, 4 (2009), 2447-2471.

A. Tartakovsky and P. Meakin. 2005. Modeling of surface tension and contact angles with smoothed particle hydrodynamics. Phys Rev E 72 (Aug 2005), 026301. Issue 2. https://doi.org/10.1103/PhysRevE.72.026301

D. Terzopoulos, J. Platt, and K. Fleischer. 1991. Heating and melting deformable models. The fournal of Visualization and Computer Animation 2, 2 (1991), 68-73.

J. Thomson. 1855. XLII. On certain curious motions observable at the surfaces of wine and other alcoholic liquors. London, Edinburgh, and Dublin Phil Mag 7 Sci 10, 67 (1855), 330-333.

N. Thürey, C. Wojtan, M. Gross, and G. Turk. 2010. A multiscale approach to mesh-based surface tension flows. ACM Trans Graph (TOG) 29, 4 (2010), 1-10.

M. Tong and D. J. Browne. 2014. An incompressible multi-phase smoothed particle hydrodynamics (SPH) method for modelling thermocapillary flow. Int $\mathcal{f}$ Heat Mass Transfer 73 (2014), 284 - 292. https://doi.org/10.1016/j.ijheatmasstransfer.2014.01. 064

D. C. Venerus and D. N. Simavilla. 2015. Tears of wine: New insights on an old phenomenon. Scientific reports 5 (2015), 16162.

C. Wang, Q. Zhang, H. Xiao, and Q. Shen. 2012. Simulation of multiple fluids with solidliquid phase transition. Comp Anim Virtual Worlds 23, 3-4 (2012), 279-289. https://doi org/10.1002/cav.1457 arXiv:https://onlinelibrary.wiley.com/doi/pdf/10.1002/cav.1457

H. Wang, Y. Jin, A. Luo, X. Yang, and B. Zhu. 2020a. Codimensional Surface Tension Flow Using Moving-Least-Squares Particles. ACM Trans Graph 39, 4, Article 42 (July 2020), 16 pages. https://doi.org/10.1145/3386569.3392487

H. Wang, G. Miller, and G. Turk. 2007. Solving General Shallow Wave Equations on Surfaces (SCA '07). Eurographics Association, Goslar, DEU, 229-238.

H. Wang, P. J. Mucha, and G. Turk. 2005. Water Drops on Surfaces. ACM Tran. Graph 24, 3 (July 2005), 921-929. https://doi.org/10.1145/1073204.1073284

S. Wang, M. Ding, T. Gast, L. Zhu, S. Gagniere, C. Jiang, and J. Teran. 2019. Simulation and Visualization of Ductile Fracture with the Material Point Method. Proceedings of the ACM on Computer Graphics and Interactive Techniques 2, 2, 18.

X. Wang, M. Li, Y. Fang, X. Zhang, M. Gao, M. Tang, D. Kaufman, and C. Jiang. 2020b. Hierarchical optimization time integration for CFL-rate MPM stepping. ACM Trans Graph (TOG) 39, 3 (2020), 1-16.

X. Wang, Y. Qiu, S.R. Slattery, Y. Fang, M. Li, S.-C. Zhu, Y. Zhu, M. Tang, D. Manocha and C. Jiang. 2020c. A Massively Parallel and Scalable Multi-GPU Material Point Method. ACM Trans Graph 39, 4, Article 30 (July 2020), 15 pages. https://doi.org/ $10.1145 / 3386569.3392442$

C. Wojtan, N. Thürey, M. Gross, and G. Turk. 2010. Physics-inspired topology changes for thin fluid features. ACM Trans Graph 29, 4 (2010), 50:1-50:8. https://doi.org/10. $1145 / 1778765.1778787$

J. Wolper, Y. Chen, M. Li, Y. Fang, Z. Qu, J. Lu, M. Cheng, and C. Jiang. 2020. AnisoMPM animating anisotropic damage mechanics. ACM Trans. Graph. 39, 4, Article 37 (2020).

J. Wolper, Y. Fang, M. Li, J. Lu, M. Gao, and C. Jiang. 2019. CD-MPM: continuum damage material point methods for dynamic fracture animation. ACM Trans. Graph. 38, 4 Article 119 (2019)

S. Yang, X. He, H. Wang, S. Li, G. Wang, E. Wu, and K. Zhou. 2016a. Enriching SPH simulation by approximate capillary waves. In Symp Comp Anim. 29-36.

T. Yang, J. Chang, M. C. Lin, R. R. Martin, J. J. Zhang, and S.-M. Hu. 2017. A unified particle system framework for multi-phase, multi-material visual simulations. ACM Transactions on Graphics (TOG) 36, 6 (2017), 224.

T. Yang, M. C. Lin, R. R. Martin, J. Chang, and S.-M. Hu. 2016b. Versatile Interactions at Interfaces for SPH-Based Simulations (SCA '16). Eurographics Association, Goslar, DEU, 57-66.

T. Young. 1805 III. An essay on the cohesion of fluids Phil Tran Royal Soc London 95 (1805), 65-87. https://doi.org/10.1098/rstl.1805.0005 arXiv:https://royalsocietypublishing.org/doi/pdf/10.1098/rstl.1805.0005

Y. Yue, B. Smith, C. Batty, C. Zheng, and E. Grinspun. 2015. Continuum foam: material point method for shear-dependent flows. ACM Trans Graph 34, 5 (2015), 160:1-160:20.

T. Zhang, J. Shi, C. Wang, H. Qin, and C. Li. 2017. Robust Gas Condensation Simulation with SPH based on Heat Transfer. In Pacific Graphics Short Papers, Jernej Barbic Wen-Chieh Lin, and Olga Sorkine-Hornung (Eds.). The Eurographics Association, 27-32. https://doi.org/10.2312/pg.20171321

W. Zheng, B. Zhu, B. Kim, and R. Fedkiw. 2015. A new incompressibility discretization for a hybrid particle MAC grid representation with surface tension. 7 Comp Phys
280 (2015), 96-142.

B. Zhu, E. Quigley, M. Cong, J. Solomon, and R. Fedkiw. 2014. Codimensional surface tension flow on simplicial complexes. ACM Trans Graph (TOG) 33, 4 (2014), 1-11. 